\title{
Asymptotics for Duration-Driven Long Range Dependent Processes
}

\author{
Mengchen Hsieh* $\quad$ Clifford M. Hurvich* $\quad$ Philippe Soulier ${ }^{\dagger}$
}

November 13, 2018

\begin{abstract}
We consider processes with second order long range dependence resulting from heavy tailed durations. We refer to this phenomenon as duration-driven long range dependence (DDLRD), as opposed to the more widely studied linear long range dependence based on fractional differencing of an iid process. We consider in detail two specific processes having DDLRD, originally presented in Taqqu and Levy (1986), and Parke (1999). For these processes, we obtain the limiting distribution of suitably standardized discrete Fourier transforms (DFTs) and sample autocovariances. At low frequencies, the standardized DFTs converge to a stable law, as do the standardized sample autocovariances at fixed lags. Finite collections of standardized sample autocovariances at a fixed set of lags converge to a degenerate distribution. The standardized DFTs at high frequencies converge to a Gaussian law. Our asymptotic results are strikingly similar for the two DDLRD processes studied. We calibrate our asymptotic results with a simulation study which also investigates the properties of the semiparametric log periodogram regression estimator of the memory parameter.
\end{abstract}

JEL Classification: C14; C22

Keywords: Long Memory, Heavy Tails, Sample Autocovariances, Discrete Fourier Transform.

\section{Introduction}

The renewal-reward process of Taqqu and Levy (1986) and the error duration model of Parke (1999) are nonlinear models with long memory. Both models embody useful features not shared by traditional linear long-memory models (such as ARFIMA), in that they both allow simultaneously for regime switching and long memory without requiring these two commonly-observed phenomena to be separately parameterized. The models intrinsically possess both structural

\footnotetext{
${ }^{*}$ New York University

${ }^{\dagger}$ Université Paris X

We thank the Editor and two referees for their careful reading of the paper and for their constructive comments.
} 
change and long memory, in an inextricably intertwined manner, and thus may help practitioners to view these two phenomena as a duality rather than a dichotomy.

In the renewal-reward process, the value of the process stays constant at some random level throughout regimes of durations governed by a sequence of i.i.d. random variables with finite mean but infinite variance. In the model of Parke (1999), the process is written as a sum of present and past shocks, where shocks survive in the sum for durations governed by a long-tailed i.i.d. sequence. In both models, there is a one-to-one correspondence between the tail index of the i.i.d. duration sequence and the memory parameter of the process. Therefore, we will say that both of these models possess duration-driven long range dependence (DDLRD).

Liu (2000) has used the renewal-reward process in a stochastic volatility model for financial returns exhibiting simultaneous long memory and regime switching in the volatility. The economic motivation for such a model is a scenario where trading is stimulated by news arrivals, and the duration of a volatility regime created by a given news event is heavy-tailed. Liu (2000) fitted a such a stochastic volatility model to the returns on the S\&P 500 and found that it was successful at simultaneously capturing the long memory and heavy-tailed regime switching of volatility, and that it was successful at forecasting volatility.

The error duration model of Parke (1999) has drawn considerable recent attention among practitioners in finance and economics. By focusing on the duration of shocks rather than on fractional differencing of the shocks, the model provides an appealing paradigm for long memory in economic time series and in volatility of financial series. For example, Bollerslev and Jubinski (1999) invoked Parke's error duration mechanism to argue that under certain reasonable assumptions on the duration of the impact of particular news events, the aggregate information arrival process will have long memory, a conclusion that supports a version of the Mixture of Distributions Hypothesis (MDH). Another relevant example was given by Parke (1999), who argued that the error duration model provides a plausible mechanism for explaining long memory in aggregate employment. He assumed that an error represents the effect of a given firm on aggregate employment and holds constant for the lifetime of the firm. He then analyzed survival rates for U.S. businesses, and showed that the rates were consistent with an error duration model that induces long memory in employment.

As models possessing DDLRD gain increasing application, practitioners may feel that, if faced with data generated by a model having DDLRD, they can safely use the standard methods of data analysis and statistical inference for long-memory series. In particular, they may wish to examine the sample autocovariances, or to construct the log-periodogram regression estimator (GPH) of the memory parameter, due to Geweke and Porter-Hudak (1983), or to use the Gaussian semiparametric estimator (GSE) of Künsch (1987). Some caution may be in order here, however, since most of the existing theory assumes that the series is either Gaussian (see Robinson 1995a and Hurvich, Deo and Brodsky 1998 for GPH), linear in an i.i.d. sequence (see Velasco 2000 for GPH), linear in a Martingale difference sequence (See Robinson 1995b for GSE, Chung 2002 for autocovariances), or, in the case of volatility, that the observations can be transformed into a sum of linear series (see Deo and Hurvich 2001, Hurvich and Soulier 2002, for GPH applied to long memory stochastic volatility models). If the Taqqu-Levy and Parke models 
are to be widely accepted and used, it is necessary to build a theory for the currently-standard methodology of long-memory data analysis and inference that applies to such series. The present paper represents a first step in that direction. We will explore the asymptotic properties of the discrete Fourier transforms (DFTs) and sample autocovariances from both processes. Some of the results are surprising, and tend to confirm that caution was indeed warranted.

One surprising result we find is that both the sample autocovariances at a fixed lag and the DFT at a fixed Fourier frequency, if suitably standardized, have limiting non-Gaussian stable distributions. This implies that a data analysis based on examination of the sample autocovariances may be misleading. It also implies that data analytic methods that rely on the very low frequency behavior of the DFT of a series with DDLRD will not have the same asymptotic properties as in the linear long-memory case. (See, e.g., Chen and Hurvich (2003 a,b) on fractional cointegration of linear processes). On a more positive note, but still surprisingly, we find for the DFT at the $j$ 'th Fourier frequency $x_{j}=2 \pi j / n$ where $n$ is the sample size, that if $j$ tends to $\infty$ sufficiently quickly, then the DFT is asymptotically normal. This indicates that the DFT at not-too-low frequencies has some robustness to the type of long-memory generating mechanism. It also suggests that standard estimation methods such as GPH and GSE may retain the same properties that they are already known to have in the linear case, although some trimming of very low frequencies may be needed. Our theoretical results will be augmented with a Monte Carlo study, both to calibrate the finite-sample applicability of our theorems, and to briefly explore the properties of the GPH estimator for models with DDLRD, a topic which we do not attempt to handle theoretically here.

The organization of the remainder of this paper is as follows. In Section 2, we review some of the existing theory on second order long memory processes, so as to contrast it with the theory we will develop for the DDLRD processes. In Section 3, we give the precise formulation of the TaqquLevy and Parke models, exhibit their autocovariance functions, present a proposition which shows that Parke's process is well defined only in the stationary case, elaborate further on the differences between the models, and present some basic theory for the two models. In particular, in Section 3.4 we consider the weak convergence of partial sums for both processes, and in Section 3.5 we consider asymptotics for the empirical process in the Taqqu-Levy case. In Section 4, we present the asymptotics for the discrete Fourier transforms for both series, treating the cases of low frequencies and high frequencies separately, as the limiting distribution is different in these two cases. In Section 5, we consider the asymptotics for the sample autocovariances of the Parke and Taqqu-Levy processes. Interestingly, the joint limiting distribution of a collection of standardized sample autocovariances at a fixed finite set of lags is degenerate. In Section 6 we present the results of a simulation study. In Section 7, we present some concluding remarks. In the Appendix, we present some useful lemmas, and give the proofs of our main results.

\section{Second order long memory}

We start by recalling some classical definitions and facts about long memory processes. A second order stationary process $X=\left\{X_{t}, t \in \mathbb{Z}\right\}$ is usually said to be long range dependent if 
its autocovariance function $\gamma(t)=\operatorname{cov}\left(X_{0}, X_{t}\right)$ is not absolutely summable. This definition is too wide to be useful. A more practical condition is that the autocovariance is regularly varying: there exist $H \in(1 / 2,1)$ and a function $L$, slowly varying function at $\infty$, such that

$$
\gamma(t)=L(t)|t|^{2 H-2}
$$

A function $L$ is slowly varying at $\infty$ if it is bounded on finite intervals and if $L(a t) / L(t) \rightarrow 1$ as $t \rightarrow \infty$ for all $a>0$. For fractional Gaussian noise (i.e., the increments of a fractional Brownian motion) $L(t)$ is a positive constant. For the $\operatorname{ARFIMA}(p, d, q)$ model of Granger and Joyeux (1980), Hosking (1981), $L(t)$ approaches a positive constant as $t \rightarrow \infty$. Other examples of functions $L$ that are slowly varying at $\infty$ include $\log t$, powers of $\log t$, and iterated logarithms. For more details, see Resnick (1987) or Bingham, Goldie and Teugels (1989). Since the autocovariances of the processes considered in this paper are generated by tail probabilities of positive random variables, our general regular variation assumption is needed to allow our theory to cover cases of practical interest.

Under condition (2.1), it holds that:

$$
\lim _{n \rightarrow \infty} n^{-2 H} L(n)^{-1} \operatorname{var}\left(\sum_{t=1}^{n} X_{t}\right)=-4 \Gamma(-2 H) \cos (\pi H) .
$$

A second order stationary process satisfying (2.2) will be referred to as a second order long memory process, and the coefficient $H$ is the long memory parameter of the process, often referred to as the Hurst coefficient of the process $X$. We will henceforth use this terminology.

A weakly stationary process with autocovariance function satisfying (2.1) has a spectral density, i.e. there exists a function $f$ such that

$$
\gamma(t)=\int_{-\pi}^{\pi} f(x) \mathrm{e}^{\mathrm{i} t x} d x
$$

The function $f$ is the sum of the series

$$
\frac{1}{2 \pi} \sum_{t \in \mathbb{Z}} \gamma(t) \mathrm{e}^{-\mathrm{i} t x}
$$

which converges uniformly on the compact subsets of $[-\pi, \pi] \backslash\{0\}$ and in $L^{1}([-\pi, \pi], d x)$. It is then well known that the behaviour of the function $f$ at zero is related to the rate of decay of $\gamma$. More precisely, if we assume in addition that $L$ is ultimately monotone, we obtain the following Tauberian result:

$$
\lim _{x \rightarrow 0} L(1 / x)^{-1} x^{2 H-1} f(x)=\pi^{-1} \Gamma(2 H-1) \sin (\pi H) .
$$

(Cf. for instance Taqqu (2003), Proposition 4.1). The usual tools of statistical analysis of weakly stationary processes are the empirical autocovariance function, the discrete Fourier transform 
(DFT) and the periodogram. We will focus here on the DFT and periodogram ordinates of a sample $X_{1}, \ldots, X_{n}$, defined as

$$
d_{X, k}=(2 \pi n)^{-1 / 2} \sum_{t=1}^{n} X_{t} \mathrm{e}^{\mathrm{i} t x_{k}}, \quad I_{X, k}=\left|d_{X, k}\right|^{2},
$$

for integers $k, 1 \leq k<n / 2$. In the classical weakly stationary short memory case (when the autocovariance function is absolutely summable), it is well known that the periodogram is an asymptotically unbiased estimator of the spectral density. This is no longer true for second order long memory processes. Hurvich and Beltrao (1993) showed (assuming that $L(1 / x)$ is continuous at $x=0$, though the extension is straightforward) that for any fixed positive integer $k$, there exists a constant $c(k, H)>0$ such that

$$
\lim _{n \rightarrow \infty} \mathbb{E}\left[I_{X, k} / f\left(x_{k}\right)\right]=c(k, H) .
$$

The previous results hold for any second order long memory process. We now describe some weak convergence results that are valid for Gaussian or linear processes.

If $X$ is a second order long memory Gaussian process, then $L(n)^{-1 / 2} n^{-H} \sum_{k=1}^{[n t]} X_{k}$ converges weakly to the fractional Brownian motion $B_{H}(t)$ which is the zero mean Gaussian process with covariance function given by:

$$
\mathbb{E}\left[B_{H}(s) B_{H}(t)\right]=\frac{1}{2}\left(|s|^{2 H}-|t-s|^{2 H}+|t|^{2 H}\right) .
$$

Here weak convergence is in the space $\mathcal{D}$ of right-continuous and left-limited (càdlàg) functions on $[0, \infty)$.

This result can be extended to a strict sense linear process, i.e. a process $X$ for which there exists a sequence $\left(\epsilon_{j}\right)_{j \in \mathbb{Z}}$ of i.i.d. random variables with zero mean and finite variance, and a square summable sequence of real numbers $\left(a_{j}\right)_{j \in \mathbb{Z}}$ such that for all $t \in \mathbb{Z}$,

$$
X_{t}=\sum_{j \in \mathbb{Z}} a_{j} \epsilon_{t-j} .
$$

If $a_{j}=L(j)|j|^{H-3 / 2}$, then $X$ is a second order long memory process with Hurst coefficient $H$, and $L(n)^{-1 / 2} n^{-H} \sum_{k=1}^{[n t]} X_{k}$ converges weakly, in the sense of weak convergence of finite dimensional distributions, to the fractional Brownian motion $B_{H}(t)$. This can be proved easily by applying the Central Limit Theorem for linear processes of Ibragimov and Linnick (1971, Theorem 18.6.4). Weak convergence in the space $\mathcal{D}$ can also be proved. Cf. Gorodeckii (1977) or Lang and Soulier (2000). A classical example of such a long memory linear process is the $\operatorname{ARFIMA}(p, d, q)$ process, whose Hurst coefficient is $H=1 / 2+d$.

For Gaussian and linear processes, a weak convergence result can also be obtained for the periodogram and the DFT ordinates. For any fixed $j, f\left(x_{j}\right)^{-1 / 2} d_{X, j}$ converges to a complex Gaussian distribution with dependent real and imaginary parts. Cf. Terrin and Hurvich (1994), Chen and Hurvich (2003 a,b), Walker (2000), and Lahiri (2003). 
The asymptotic behaviour described above is different from the behaviour of weakly dependent processes, such as sequences of i.i.d. or strongly mixing random variables, whose partial sum process, renormalised by the usual rate $\sqrt{n}$, converges to the standard Brownian motion. But these long memory processes share with weakly dependent processes the Gaussian limit and the fact that weak limits and $L^{2}$ limits have consistent normalisations, in the sense that, if $\xi_{n}$ denotes one of the statistics considered above, there exists a sequence $v_{n}$ such that $v_{n} \xi_{n}$ converges weakly to a non degenerate distribution and $v_{n}^{2} \mathbb{E}\left[\xi_{n}^{2}\right]$ converges to a positive limit (which is the variance of the asymptotic distribution).

In the sequel we define two second order stationary models, which possess properties (2.1) and (2.3), but whose weak limit behaviour is extremely different from that of Gaussian or linear models. In Section 3 we define these models.

\section{Formulation of the Models}

\subsection{The Taqqu-Levy Model}

Let $\left\{T_{k}\right\}$ be i.i.d. positive integer-valued random variables with mean $\mu$, in the domain of attraction of a stable distribution with tail index $\alpha \in(1,2)$, i.e. there exists a function $L$, slowly varying at infinity such that for all $n \geq 1$,

$$
\mathbb{P}\left(T_{1} \geq n\right)=L(n) n^{-\alpha}
$$

To avoid trivialities, we also assume that $\mathbb{P}\left(T_{1}=1\right)>0$. Let $S_{0}$ be a non-negative integer-valued random variable, independent of the $\left\{T_{k}\right\}$, with probability distribution

$$
P\left(S_{0}=u\right)=\mu^{-1} P\left(T_{k} \geq u+1\right), u=0,1, \ldots
$$

Let $\left\{W_{k}\right\}$ be i.i.d. random variables with $E\left[W_{k}\right]=0$ and $\operatorname{var}\left[W_{k}\right]=\sigma_{W}^{2}<\infty$. Assume that the $\left\{W_{k}\right\}$ are independent of $S_{0}$ and $\left\{T_{k}\right\}$. We observe a process denoted by $\left\{X_{t}\right\}$ for $t=1, \ldots, n$. The observed process is constant on regimes (intervals) determined by $S_{0}$ and the interarrival times $T_{k}$. The constant value on each regime is given by one of the $\left\{W_{k}\right\}$. The time between the start of the sample and the first change of regime is $S_{0}$, and the subsequent waiting times are $T_{1}, T_{2}, \ldots$. The total time up to the end of the $k^{\prime}$ th regime $(k=0,1, \ldots)$ is given by $S_{-1} \equiv-1$, $S_{0}$ and

$$
S_{k}=S_{0}+T_{1}+\ldots+T_{k}, k=1,2, \ldots
$$

The observed process $\left\{X_{t}\right\}$ is given by $W_{k}$ if $t$ lies in the $k$ 'th regime, so that

$$
X_{t}=\sum_{k=0}^{\infty} W_{k} \mathbf{1}_{\left\{S_{k-1} \leq t<S_{k}\right\}},
$$

where $\mathbf{1}_{A}$ is the indicator function of the set $A$. Let $M_{n}$ be the counting process associated with the renewal process $\left\{S_{0}, S_{1}, \ldots\right\}$, i.e. a non-negative integer-valued random variable denoting 
the total number of regime changes in the series before the time $n-1$ :

$$
M_{n}=k \Leftrightarrow S_{k-1} \leq n<S_{k}
$$

The renewal process $\left\{S_{0}, S_{1}, \ldots\right\}$ is called a stationary renewal process, in the sense that the counting process $M_{n}$ has stationary increments, whence the following result (Cf. Liu 2000, Theorem 1.1).

Proposition 3.1. The process $X$ defined by (3.3) is strictly stationary with zero mean and covariances

$$
\operatorname{cov}\left(X_{0}, X_{r}\right)=\sigma_{W}^{2} \mathbb{P}\left(S_{0} \geq r\right)=\mu^{-1} \sigma_{W}^{2} \mathbb{E}\left[\left(T_{1}-r\right) \mathbf{1}_{\left\{T_{1} \geq r\right\}}\right] .
$$

If (3.1) holds with $1<\alpha<2$ and $L$ ultimately monotone, then $X$ is second order long memory with Hurst coefficient $H=(3-\alpha) / 2$ and spectral density $f$ satisfying

$$
\lim _{x \rightarrow 0} L(1 / x)^{-1} x^{2 H-1} f(x)=\frac{\sigma_{W}^{2}}{2 \pi(1-H) \mu} \Gamma(2 H-1) \sin (\pi H) .
$$

\subsection{The Parke Model}

Let $\left(\epsilon_{t}\right)_{t \in \mathbb{Z}}$ be a sequence of i.i.d. random variables with zero mean and $\left(n_{s}\right)_{s \in \mathbb{Z}}$ be a sequence of i.i.d. non-negative integer valued random variables which is independent of $\left(\epsilon_{t}\right)_{t \in \mathbb{Z}}$. For $s \in \mathbb{Z}$, define

$$
g_{s, t}=1 \Leftrightarrow s \leq t \leq s+n_{s} .
$$

Parke's error duration process is then defined as:

$$
X_{t}=\sum_{s \leq t} g_{s, t} \epsilon_{s}
$$

Let $N$ be a generic random variable with the same distribution as the $n_{s}$, and define

$$
p_{k}:=\mathbb{P}(N \geq k) \quad k \geq 0 .
$$

$\left(p_{k}\right)_{k \geq 0}$ is then a non-increasing sequence such that $p_{0}=1$ and $\lim _{k \rightarrow \infty} p_{k}=0$.

Parke (1999) does not discuss the existence of this process. In his main result, he assumes that it is well defined and second order stationary. Since the terms in the sum defining the process are not vanishing, by well defined we mean that the sum is almost surely finite. We now give a necessary and sufficient condition for the process $X$ to be well defined.

Proposition 3.2. Parke's process is well defined if and only if $\mathbb{E}[N]<\infty$. In that case it is strictly stationary. If moreover $\epsilon_{0}$ has variance $\sigma_{\epsilon}^{2}$, then Parke's process has mean zero, finite variance and covariances

$$
\operatorname{cov}\left(X_{0}, X_{r}\right)=\sigma_{\epsilon}^{2} \sum_{j \geq r} p_{j}=\sigma_{\epsilon}^{2} \mathbb{E}\left[(N+1-r) \mathbf{1}_{\{N \geq r\}}\right]=\sigma_{\epsilon}^{2} \sum_{k=r}^{\infty} p_{k} .
$$


If the survival probabilities $p_{k}$ are regularly varying with index $\alpha \in(1,2)$, i.e. if they satisfy

$$
p_{j}=\mathbb{P}(N \geq j)=L(j) j^{-\alpha}, \quad j \geq 1,
$$

where $L$ is slowly varying and ultimately monotone at infinity, then Parke's error duration model $X$ exhibits second order long memory with Hurst coefficient $H=(3-\alpha) / 2$ and its spectral density $f$ satisfies

$$
\lim _{x \rightarrow 0} L(1 / x)^{-1} x^{2 H-1} f(x)=\frac{\sigma_{\epsilon}^{2}}{2 \pi(1-H)} \Gamma(2 H-1) \sin (\pi H) .
$$

\subsection{Differences between the two models}

In the error duration process, the durations are independent of birth dates which are deterministic and shocks can overlap; in the renewal-reward process, the durations are exactly equal to the interval between two consecutive birth dates, which form a renewal process, so that there is only one surviving reward at any given time point, and it is precisely the value of the process. In both cases long memory is caused by the heavy-tailedness of the durations. This property implies that some of the durations are eventually extremely long, as illustrated in Figures 1 and 2 .

Variations on these processes, which retain their main features, are possible; see Deo, Hsieh, Hurvich and Soulier (2006) for a detailed account. Given the econometric motivation of the error duration and renewal-reward processes given by Parke (1999) and Liu (2000), and since it would be difficult to present a completely unified presentation and proof of our results, we consider in this paper only these two processes.

In Figure 1, the horizontal lines represent the durations $n_{s}$ of the shocks $\epsilon_{s}$. The value of the error duration process (the bullet) at some time point is the sum of present and past shocks. The shock $\epsilon_{t-2}$ lasts for a very long time and is still present at time $t+1$, whereas the shock $\epsilon_{t-1}$ exists only over one period of time.

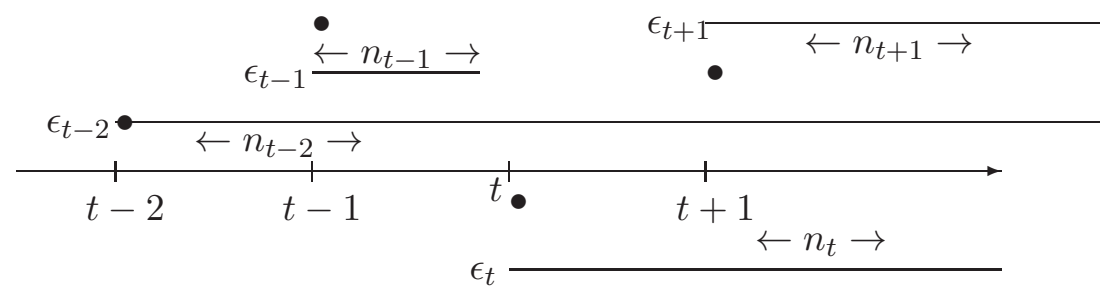

Figure 1: A path $(\bullet)$ of the error duration process: $X_{t}=\epsilon_{t-2}+\epsilon_{t}, X_{t+1}=\epsilon_{t-2}+\epsilon_{t}+\epsilon_{t+1}$.

In Figure 2, a portion of a path of the renewal-reward process is drawn; the interval $T_{k+1}$ is very long, so that the process $X$ is constant over a long period of time between $S_{k}$ and $S_{k+1}$. 


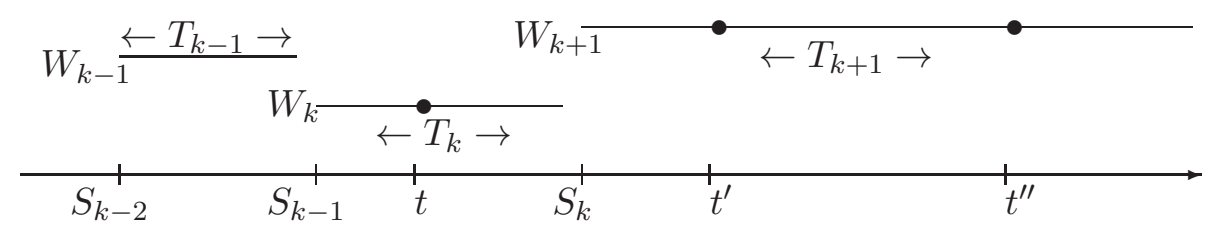

Figure 2: A path $(\bullet)$ of the renewal-reward process: $X_{t}=W_{k}, X_{t^{\prime}}=X_{t^{\prime \prime}}=W_{k+1}$.

\subsection{Asymptotics For Partial Sums}

Let $X$ denote either Parke's or Taqqu-Levy's process. The next proposition shows that although the process $X$ is second order stationary and its autocovariance function exhibits long range dependence, the partial sum process of $X$ converges to a stable Lévy process with independent increment, which implies that its behaviour mimics that of a sum of i.i.d. heavy tailed random variables. In the case of the Taqqu-Levy Process, it is stated without proof in Taqqu and Levy (1986); it can also be seen as a particular case of Theorem 2 in Mikosch et al. (2002).

Proposition 3.3. Assume that (3.1) and (3.4) hold with $1<\alpha<2$. Denote $\ell(n)=n^{-1 / \alpha}$ inf $\left\{t>0: \mathbb{P}(U>t)<n^{-1}\right\}$ with $U=T_{1}$ for the Taqqu-Levy process and $U=N$ for the Parke process. Then the finite dimensional distributions of $\ell(n)^{-1} n^{-1 / \alpha} \sum_{k=1}^{[n t]} X_{k}$ converge weakly to those of the $\alpha$-stable Levy process $\Lambda_{\alpha}$ with characteristic function

$$
\mathbb{E}\left[\mathrm{e}^{\mathrm{i} u \Lambda_{\alpha}(t)}\right]=\exp \left\{-t|u|^{\alpha} \mu^{-1} \mathbb{E}\left[|\xi|^{\alpha}\right] \Gamma(1-\alpha) \cos (\pi \alpha / 2)(1-\mathrm{i} \beta \operatorname{sign}(\mathrm{u}) \tan (\pi \alpha / 2))\right\},
$$

with $\beta=\left(\mathbb{E}\left[\xi_{+}^{\alpha}\right]-\mathbb{E}\left[\xi_{-}^{\alpha}\right]\right) / \mathbb{E}\left[|\xi|^{\alpha}\right]$ and $\xi=W_{1}$ in the case of Taqqu-Levy's process and $\xi=\epsilon_{1}$ and $\mu=1$ in the case of Parke's process.

\subsection{Empirical process of Taqqu-Levy's process}

In the case of Taqqu-Levy's process, the invariance principle (i.e., the limit theorem for renormalized partial sums) can be straightforwardly extended to an invariance principle for instantaneous functions of the process: if $\phi$ is a measurable function such that $\mathbb{E}\left[\phi^{2}\left(W_{1}\right)\right]<\infty$ and $\mathbb{E}\left[\phi\left(W_{1}\right)\right]=0$, then the finite dimensional distributions of $\ell(n)^{-1} n^{-1 / \alpha} \sum_{k=1}^{[n t]} \phi\left(X_{k}\right)$ converge weakly to those of an $\alpha$-stable Levy process, where $\ell$ is the same slowly varying function as in proposition 3.3. For Parke's process, we conjecture that this is true for polynomial functions. It is actually shown in the case $\phi(x)=x^{2}-\mathbb{E}\left[\epsilon_{1}^{2}\right]$ in Theorem [5.1, and a similar proof would probably work in the case of a higher order polynomial.

In the special case of an indicator function, we obtain the usual interval-indexed empirical process:

$$
\hat{F}_{n}(x)=\frac{1}{n} \sum_{k=1}^{n} \mathbf{1}_{\left\{X_{k} \leq x\right\}} .
$$


Let $F_{W}(x)=\mathbb{P}\left(W_{1} \leq x\right)$ be the distribution function of $W_{1}$. Then $\hat{F}_{n}$ is an estimator of $F_{W}$ and we have the following convergence result.

Theorem 3.1. The finite dimensional distributions of the process $\ell(n)^{-1} n^{1-1 / \alpha}\left(\hat{F}_{n}-F_{W}\right)$ converges weakly to those of the process $\Lambda_{\alpha}(F(\cdot))$, where $\Lambda_{\alpha}$ is the stable Levy process with characteristic function

$$
\mathbb{E}\left[\mathrm{e}^{\mathrm{i} u \Lambda_{\alpha}(t)}\right]=\exp \left\{-t|u|^{\alpha} \mu^{-1} \Gamma(1-\alpha) \cos (\pi \alpha / 2)(1-\mathrm{i} \operatorname{sign}(\mathrm{u}) \tan (\pi \alpha / 2))\right\},
$$

and $\ell(n)=n^{1 / \alpha} \inf \left\{t>0: \mathbb{P}\left(T_{1}>t\right)<n^{-1}\right\}$.

\section{Asymptotics for the DFTs}

Define

$$
D_{n, j}=\sum_{t=1}^{n} X_{t} \mathrm{e}^{\mathrm{i} t x_{j}},
$$

where $X$ denotes either Taqqu-Levy's or Parke's process.

\subsection{Low frequencies}

Proposition 4.1. Define $d_{n, j}=\sum_{k=1}^{[n / \mu]} \eta_{k} \mathrm{e}^{\mathrm{i} \mu k x_{j}}$ with $\eta_{k}=T_{k} W_{k}$ for the Taqqu-Levy process and $\eta_{k}=n_{k} \epsilon_{k}$ and $\mu=1$ for Parke's process. If (3.1) and (3.4) hold and if $j \leq n^{\rho}$ for some $\rho \in(0,1-1 / \alpha)$, then $\ell(n)^{-1} n^{-1 / \alpha}\left(D_{n, j}-d_{n, j}\right)=o_{P}(1)$, where $\ell$ is defined as in Proposition 3.3 .

Since in both cases $\eta_{k}$ belongs to the domain of attraction of an $\alpha$-stable law, Proposition 4.1 implies if $j \leq n^{\rho}$, then $\ell(n)^{-1} n^{-1 / \alpha} D_{n, j}$ converges to a stable distribution. The conclusion of Proposition 4.1 continues to hold if the upper bound $n^{\rho}$ is replaced by $c n^{\rho}$, where $c$ is a positive constant. In the case of fixed frequencies, we can describe more precisely the asymptotic distribution of the suitably normalized DFT coefficients.

Theorem 4.1. Let $j_{1}<\cdots<j_{q}$ be $q$ fixed positive integers. Let $\ell$ be defined as in Proposition 3.3. Then $\ell(n)^{-1} n^{-1 / \alpha}\left(D_{n, j_{1}}, \ldots, D_{n, j_{q}}\right)$ converge in law to the complex $\alpha$-stable vector $\left(\int_{0}^{1} \mathrm{e}^{2 \mathrm{i} \pi j_{1} s} d \Lambda_{\alpha}(s), \ldots, \int_{0}^{1} \mathrm{e}^{2 \mathrm{i} \pi j_{q} s} d \Lambda_{\alpha}(s)\right)$, where $\Lambda_{\alpha}$ is the $\alpha$-stable Levy process with characteristic function given by (3.5).

\subsection{High frequencies}

In the high frequency case, the asymptotic behaviour of the discrete Fourier transform is the same as it is for linear series. 
Theorem 4.2. If let $j$ be a non decreasing sequence of integers such that $j / n \rightarrow 0$ and $j \geq n^{\rho}$ for some $\rho \in(1-1 / \alpha, 1)$, then $\left(2 \pi n f\left(x_{j}\right)\right)^{-1 / 2} D_{n, j}$ is asymptotically complex Gaussian with independent real and imaginary parts, which are each zero mean Gaussian with variance 1/2.

The conclusion of Theorem 4.2 continues to hold if the lower bound $n^{\rho}$ is replaced by $c n^{\rho}$, where $c$ is a positive constant.

\section{Asymptotics for the Sample ACF}

The empirical autocovariance is often used as a diagnostic for long memory, hence it is of importance to investigate its properties in the present context. For $n \geq 1$ and $k \geq 0$, define $\bar{X}_{n}=n^{-1} \sum_{k=1}^{n} X_{k}$ and

$$
\hat{\gamma}_{n}(k)=n^{-1} \sum_{t=1}^{n-k}\left(X_{t}-\bar{X}_{n}\right)\left(X_{t+k}-\bar{X}_{n}\right) .
$$

Since in both cases, $X$ is a second order stationary process, $\hat{\gamma}_{n}(k)$ is an asymptotically unbiased estimator of $\gamma(k)=\operatorname{cov}\left(X_{0}, X_{k}\right)$. In the next proposition, we show that it is also a consistent estimator and obtain its rate of convergence and asymptotic distribution.

Theorem 5.1. Assume that (3.1) and (3.4) hold, $\mathbb{E}\left[\left|\epsilon_{0}\right|^{q}\right]<\infty$ and $\mathbb{E}\left[\left|W_{0}\right|^{q}\right]<\infty$ for some $q>2 \alpha$. Denote $\xi_{s}=W_{s}^{2}\left\{T_{s}-\mathbb{E}\left[T_{1}\right]\right\}$ or $\xi_{s}=\epsilon_{s}^{2}\left\{n_{s}-\mathbb{E}\left[n_{1}\right]\right\}$. Let $\mu=1$ in the case of Parke's process. Then for any fixed $k \geq 0$ and any slowly varying function $h$,

$$
\hat{\gamma}_{n}(k)-\gamma(k)=\frac{1}{n} \sum_{s=1}^{[n / \mu]} \xi_{s}+o_{P}\left(h(n) n^{1 / \alpha-1}\right) .
$$

Define $\ell$ as in Proposition 3.3. Then $\ell(n)^{-1} n^{1-1 / \alpha}\left(\hat{\gamma}_{n}(k)-\gamma(k)\right)$ converges weakly to an $\alpha$-stable random variable $\zeta$ with characteristic function

$$
\mathbb{E}\left[\mathrm{e}^{\mathrm{i} u \zeta}\right]=\exp \left\{-|u|^{\alpha} m_{\alpha} \Gamma(1-\alpha) \cos (\pi \alpha / 2)(1-\mathrm{i} \operatorname{sign}(\mathrm{u}) \tan (\pi \alpha / 2))\right\},
$$

with $m_{\alpha}=\mathbb{E}\left[\left|\epsilon_{1}\right|^{2 \alpha}\right]$ in the case of Parke's process and $m_{\alpha}=\mathbb{E}\left[\left|W_{1}\right|^{2 \alpha}\right] / \mu$ in the case of the Taqqu-Levy process.

Remark 5.1. The $o_{P}$ term in (5.2) is not uniform with respect to $k$, but (5.2) implies that for any fixed integers $q, k_{1}, \ldots, k_{q}$, the asymptotic distribution of the vector $\ell(n)^{-1} n^{1-1 / \alpha}\left[\hat{\gamma}_{n}\left(k_{1}\right)-\right.$ $\left.\gamma\left(k_{1}\right), \ldots, \hat{\gamma}_{n}\left(k_{q}\right)-\gamma\left(k_{q}\right)\right]$ is that of an $\alpha$-stable vector whose components are equal. Thus, the joint limiting distribution of a finite collection of standardized sample autocovariances at fixed lags is degenerate.

Remark 5.2. Since we assume that the shocks $\epsilon_{t}$ or rewards $W_{k}$ have zero mean, the process $X$ itself has zero mean for both models. If it is known to the data analyst that the mean is zero then no mean correction is needed in the sample autocovariances. However, in practice this knowledge is rarely assumed, so we have presented our results for the mean corrected version. 
Remark 5.3. The conclusions of Theorem 5.1 hold also for $\ell(n)^{-1} n^{1-1 / \alpha}\left(\hat{\rho}_{n}(k)-\rho(k)\right)$ where $\hat{\rho}_{n}(k)$ and $\rho(k)$ are the sample and population autocorrelations at lag $k$. This non-Gaussian limiting distribution (as well as the degeneracy described above) for the standardized sample autocorrelations will clearly affect the asymptotic properties of parametric method-of-moments estimators which are based on a finite number of sample autocorrelations. See, for example, Tieslau, Schmidt and Baillie (1996).

\section{Simulations}

Throughout this section, we denote the long-memory parameter by $d \in(0,0.5)$. Note that $d=H-1 / 2=1-\alpha / 2$. In all of our simulations, we use a sample size of $n=10000$. We chose to use $\operatorname{ARFIMA}(0, d, 0)$ autocovariances in our simulations because they are nonnegative and monotone non-increasing, which is consistent with the nonnegative and non-increasing autocovariances implied by both the Taqqu-Levy and Parke models. Let $\gamma(t)$ be the autocovariance sequence of an $\operatorname{ARFIMA}(0, d, 0)$ process,

$$
\gamma(t)=\frac{\Gamma(t+d) \Gamma(1-2 d)}{\Gamma(t-d+1) \Gamma(1-d) \Gamma(d)} \sigma_{0}^{2}, t=0,1, \ldots
$$

where $\sigma_{0}$ is the standard deviation of the ARFIMA innovations. For the integer-valued interarrival time $S_{0}$ as well as the $\left\{T_{k}\right\}$ in the Taqqu-Levy process and the survival times $\left\{n_{s}\right\}$ in the Parke process, we use the following simulation algorithm : Let $X$ denote either $S_{0}, T_{k}$ or $n_{s}$ and let $G(x)=P(X \geq x)$. We can simulate an observation $x$ of $X$ by drawing an observation $u$ of a uniform random variable and setting $x$ to be the integer such that

$$
G(x) \geq u>G(x+1) .
$$

In all cases we consider here, $G(x)$ is expressed in terms of the Gamma function, so that there is an easily evaluated continuous increasing function $\tilde{G}(x)$ which is equal to $G(x)$ for all integer values at which $G(x)$ is defined. The solution to (6.2) can be written as

$$
x=\left\lfloor\tilde{G}^{-1}(u)\right\rfloor,
$$

where $\lfloor x\rfloor$ denotes the greatest integer less than $x$. We obtain the solution $x$ to (6.3) using a simple bisection algorithm (see, eg, Johnson and Riess 1977 page 115).

\subsection{Simulation of Taqqu-Levy Process}

Before describing our sampling algorithm, we provide some convenient formulas for $P\left(S_{0} \geq t\right)$ and $P\left(T_{k} \geq t\right)$. From (3.2) and Proposition 3.1, we have

$$
\mu=\frac{1}{P\left(S_{0}=0\right)} \text { and } \sigma_{W}^{2}=\gamma(0)
$$


and

$$
P\left(S_{0} \geq t\right)=\frac{\gamma(t)}{\gamma(0)}, t=0,1,2, \ldots
$$

Thus, for $t \geq 1$, we have:

$$
\begin{aligned}
P\left(T_{k} \geq t\right) & =\mu P\left(S_{0}=t-1\right)=\frac{P\left(S_{0}=t-1\right)}{P\left(S_{0}=0\right)} \\
& =\frac{\mathbb{P}\left(S_{0} \geq t-1\right)-\mathbb{P}\left(S_{0} \geq t\right)}{\mathbb{P}\left(S_{0} \geq 0\right)-\mathbb{P}\left(S_{0} \geq 1\right)}=\frac{\gamma(t-1)-\gamma(t)}{\gamma(0)-\gamma(1)}
\end{aligned}
$$

For all of our simulations of the Taqqu-Levy process, we assume that $\sigma_{0}^{2}=1$. From (6.4) and (6.5), we can sample $S_{0}$ and $\left\{T_{k}\right\}$ using the bisection algorithm. We also simulate iid normal random variables $W_{k}$ with mean zero and variance $\sigma_{W}^{2}=\gamma(0)$, independent of $S_{0}$ and $\left\{T_{k}\right\}$. The duration of the 0 th regime is $S_{0}$ and the duration of the $k$ th regime is $T_{k}$ for $k \geq 1$. The value of the series $X_{t}$ is constant at $W_{k}$ throughout the $k$ th regime. This yields the simulated

realization $X_{0}, \ldots, X_{n-1}$. Occasionally, the entire simulated realization was constant, as there were no breaks before $n-1$. Such realizations were discarded.

\subsection{Simulation of Parke's Process}

By Proposition 3.2, Parke's process is well defined if and only if with probability one, for all $t$, there is a finite number of shocks surviving at time $t$. This allows us to simulate a process which is distributionally equivalent to Parke's using only a finite sum

$$
X_{t}=\sum_{s=-J}^{t} g_{s, t} \epsilon_{s}, t=1,2, \ldots
$$

where $-J$ is the time index of the oldest shock that survives at time $t=0$. The non-negative integer-valued random variable $J$ has a probability distribution

$$
P(J \leq j)=\prod_{k=j+1}^{\infty}\left(1-p_{k}\right) .
$$

In order to obtain the covariances (6.1), for $0<d<1 / 2$, the survival probabilities are defined by (see Parke, 1999)

$$
p_{k}=\frac{\Gamma(2-d)}{\Gamma(d)} \frac{\Gamma(k+d)}{\Gamma(k+2-d)}, k=0,1,2, \ldots
$$

For each realization of Parke's process, we start by sampling $J$ from the probability distribution determined by (6.7) truncated to the range $(0,1,2, \ldots, 10000)$. This was adequate for the values of $d$ considered here, $d=0.1$ and $d=0.4$, since the sum of the probabilities up to that truncation 
point is extremely close to one in both cases. Next, we generate a sequence of standard normal shocks $\left\{\epsilon_{s}\right\}_{s=-J}^{n}$. The innovation variance $\sigma_{0}^{2}$ of the $\operatorname{ARFIMA}(0, d, 0)$ process is related to $\sigma_{\epsilon}^{2}$ (we have $\sigma_{\epsilon}^{2}=1$ ) by

$$
\sigma_{0}^{2}=\frac{\Gamma(1-d) \Gamma(2-d)}{\Gamma(2-2 d)} \sigma_{\epsilon}^{2}
$$

Next we discuss the simulation of the $\left\{n_{s}\right\}$ sequence. Special attention must be paid to the survival time $n_{-J}$ for the oldest shock $\epsilon_{-J}$. It is not sampled from the probability distribution determined by $\left\{p_{k}\right\}$, but rather from the conditional distribution

$$
P(N \geq i \mid N \geq J)=\frac{p_{i}}{p_{J}}, i \geq J .
$$

We apply the bisection algorithm to sample $n_{-J}$ and the other $\left\{n_{s}\right\}_{s=-J+1}^{n}$ from (6.8) and (6.10). Using the values $\left\{n_{s}\right\}_{s=-J}^{-1}$, we compute the "death time" for each prehistoric shock $\left\{\epsilon_{s}\right\}_{s=-J}^{-1}$. At each time $t \geq 0$, there may be some past shocks dying, so the time series $X_{t}$ is generated by adding a new shock to the previous value $X_{t-1}$ and subtracting the sum of those shocks dying at time $t$.

\subsection{Simulation Results}

We performed Monte Carlo simulations to assess the finite sample properties of the DFT coefficients in light of Theorems 4.1 and 4.2 for both the Taqqu-Levy and Parke processes. We generated 500 replications of length $n=10000$ in each case. Recall that $d=1-\frac{1}{2} \alpha$, and $1<\alpha<2$. We used autocovariances corresponding to an $\operatorname{ARFIMA}(0, d, 0)$ model as described earlier, with $d=0.1$ and $d=0.4$. For each value of $d$, the normalized Fourier coefficients were evaluated at frequency $x_{j}$ with $j=1,2,\left\lfloor n^{0.2}\right\rfloor,\left\lfloor n^{0.4}\right\rfloor,\left\lfloor n^{0.6}\right\rfloor,\left\lfloor n^{0.8}\right\rfloor,\left\lfloor\frac{n}{2}\right\rfloor-2,\left\lfloor\frac{n}{2}\right\rfloor-1$. For the Taqqu-Levy process with $d=0.4$, there were 60 constant realizations. We excluded these constant realizations from our analysis, while keeping the number of realizations used at 500 .

Figures 34 4 present the normal Quantile-Quantile (QQ) plots of the normalized Fourier cosine coefficients $A_{j} / f\left(x_{j}\right)^{\frac{1}{2}}$ for the Parke process with $d=0.1$ and $d=0.4$, where

$$
A_{j}=\frac{1}{(2 \pi n)^{1 / 2}} \sum_{t=0}^{n-1} x_{t} \cos \left(x_{j} t\right) .
$$

The number inside the parenthesis at the bottom of each QQ plot represents the $p$-value for the Anderson-Darling test of normality. According to Theorems 4.1 and 4.2, if $j$ increases sufficiently quickly with the sample size $n$, i.e. when $j \geq n^{\rho}$ for $\rho>1-1 / \alpha$, the normalized Fourier coefficients are asymptotically normal. Furthermore, as $d$ increases, the value of $\alpha$ will decrease, and the condition on the rate of increase of $j$ to ensure asymptotic normality becomes less stringent. When $d=0.1$, we have $1-1 / \alpha=0.4444$, a number larger than $1-1 / \alpha=0.1667$ when $d=0.4$. For the Parke process with $d=0.1$, we do not reject the hypothesis of normality 
for $j \geq n^{0.4}$; while $d=0.4$, we reject the hypothesis of normality for $j<n^{0.2}$. Thus our simulation results are essentially consistent with the results of Theorems 4.1 and 4.2 . We found similar results for the Taqqu-Levy process. Since the results for the normalized Fourier sine coefficient

$$
B_{j}=\frac{1}{(2 \pi n)^{1 / 2}} \sum_{t=0}^{n-1} x_{t} \sin \left(x_{j} t\right)
$$

are very similar to those we found here, we do not present them here.

Figure 5 presents scatterplots of the average log normalized periodogram vs. $\log \left|2 \sin \left(x_{j} / 2\right)\right|$ at the Fourier frequencies from $j=1, \ldots, 4999$. We would expect a horizontal line across all frequencies if $E\left[\log \frac{I\left(x_{j}\right)}{f\left(x_{j}\right)}\right]$ is constant for all $j$. The plots indicate that at low Fourier frequencies, the average log normalized periodogram is changing but approaches a constant as $j$ increases. If $I\left(x_{j}\right) / f\left(x_{j}\right)$ were distributed as $(1 / 2) \chi_{2}^{2}$ as would be the case for a Gaussian white noise process, we would have $E\left[I\left(x_{j}\right) / f\left(x_{j}\right)\right]=-\gamma=-0.577216$ in Figure 5. There seems to be some evidence that the log normalized periodogram is biased upward for the Taqqu-Levy process with $d=0.4$, but not for the other situations considered. Note that since the DFT coefficients converge weakly to an $\alpha$ stable law at fixed low Fourier frequencies, we should expect higher variability of the log normalized periodogram at these frequencies. This suggests that if we regress $\left\{\log \left(I\left(x_{j}\right)\right)\right\}$ on $\left\{\log \left(f\left(x_{j}\right)\right)\right\}$ without trimming a set of low Fourier frequencies, we may get a biased and/or highly variable GPH estimator. Further evidence is given in Figure 6, which presents scatterplots of the average of $\log \left(I\left(x_{j}\right)\right)$ vs. $\log 2\left|\sin \left(x_{j} / 2\right)\right|$ together with their fitted least-squares lines. We also found that there are several outliers at low frequencies for both processes with $d=0.1$ as well as $d=0.4$. However, there are more outliers in the case of $d=0.1$ for both processes. The fact that the normalized periodogram behaves differently at the low Fourier frequencies may present a problem for the GPH estimator if we include all Fourier frequencies. The presence of the low outliers found above is not surprising since under DDLRD, for fixed $j, I\left(x_{j}\right) / f\left(x_{j}\right)=o_{p}(1)$ for fixed $j$ under DDLRD. See Comment 3 in Section 7.

Figure 7 presents normal QQ plots for the sample autocorrelations based on the Taqqu-Levy process with $d=0.1$. The Anderson-Darling $p$-values are extremely small so we reject the null hypothesis of normality in all cases. Furthermore, the plots indicate long-tailed distributions. These findings do not contradict Theorem 5.1 which states that the autocovariances for both processes will converge to an $\alpha$-stable law. We found similar results for the Taqqu-Levy process with $d=0.4$ as well as the Parke process for both values of $d$.

Tables 1 and 2 present simulation variances of the normalized DFT cosine coefficients and the corresponding normal-based $95 \%$ confidence intervals for the true variance, $\sigma^{2}$. We do not reject the null hypothesis that $\sigma^{2}=0.5$ for any $j$ when $d=0.1$ in the Taqqu-Levy process, but when $d=0.4$, we reject the null hypothesis for $j=n / 2-1$. For the Parke process, we accept the null hypothesis for all Fourier frequencies with both values of $d$ except for $j=n^{0.2}$ in the case $d=0.1$. Thus the results are essentially consistent with the theoretical variances stated in Theorem 4.2 . 


\section{Concluding remarks; topics for future research}

1. The main theoretical results we have obtained for the Parke and Taqqu-Levy models are strikingly similar. Also, it seems clear that the class of processes having DDLRD is much larger than the two processes we have considered in this paper. A specific example of another such process is the random coefficient autoregression studied in Leipus and Surgailis (2002). We have so far been unable to find an overarching unification for DDLRD processes which would allow the development of a single set of theoretical results that applies to the entire class, although such a unification seems desirable, and may well be possible.

2. In Robinson (1995a), the theory of a modified GPH estimator was developed for Gaussian long-memory processes. One aspect of the modification was that an increasing number of low frequencies were trimmed (omitted) before constructing the estimate. Subsequently Hurvich, Deo and Brodsky (1998), who also assumed Gaussianity, showed that trimming can be avoided. More recently, Hurvich, Moulines and Soulier (2002) showed that trimming can also be avoided in a different log-periodogram regression estimator, assuming a linear, potentially non-Gaussian series. For linear series, it is known that the DFT at fixed $j$ is asymptotically normal (Terrin and Hurvich, 1994), but that the periodogram is asymptotically neither independent, identically distributed, nor exponentially distributed (Künsch 1986, Hurvich and Beltrao 1993). Simulations, mostly from Gaussian long-memory series, indicate that trimming yields a very modest bias reduction, while inflating the variance of the GPH estimator substantially. (See also Deo and Hurvich 2001, in the context of LMSV models).

In contrast, the results of the present paper indicate that if the long memory is generated by DDLRD, then trimming of low frequencies may in fact be desirable. The DFT at fixed $j$ converges in distribution to an infinite-variance stable distribution, but (under a different normalization; see Comment 3 below) if $j$ is allowed to increase suitably quickly a limiting normal distribution results. It is unclear at this moment whether trimming is needed to establish the asymptotic normality of the GPH estimator based on a process having DDLRD, but clearly the failure to trim low frequencies may adversely affect the finitesample behavior of the GPH estimator. Paradoxically, the larger $d$ is, the less stringent the conditions on the rate of increase of $j$ to ensure asymptotic normality. This seems to indicate that when $d$ is larger less trimming would be needed, both in theory and in practice. This runs counter to the effects studied by Hurvich and Beltrao (1993) (which concern only the second order structure of the process) which imply that the bias of the normalized periodogram increases as $d$ increases from zero.

In any case, it should be stressed that we have not attempted to derive in this paper any asymptotic properties for the GPH estimator or any other estimator of the long memory parameter under DDLRD. We leave this as a topic for future research.

3. There is as yet no reliable way to distinguish between linear long memory and DDLRD on the basis of an observed data set. Some of our theoretical results may ultimately prove 
helpful in this regard, but we leave this as a topic for future research. We note here that the low-frequency periodogram ordinates, normalized by the spectral density, are asymptotically normal under linear long memory models, but converge in probability to zero under DDLRD. This latter result follows since from our Proposition 4.1, we obtain after a short calculation that for fixed $j, I\left(x_{j}\right) / f\left(x_{j}\right)=O_{p}(2 / \alpha+\alpha-3)$, so that $I\left(x_{j}\right) / f\left(x_{j}\right)=o_{p}(1)$. It also follows from Proposition 4.1 that in the DDLRD case under a different normalization, the periodogram $I\left(x_{j}\right)$ is asymptotically stable if $j$ is fixed.

4. It is known (see Chung 2002 and the references therein) that for a long-memory process linear in martingale differences, the autocovariances are asymptotically normal if $d<1 / 4$, but converge to a non-normal, finite-variance distribution if $d \in(1 / 4,1 / 2)$. So the asymptotics for the sample autocovariances depend on $d$, which is an undesirable property from the point of view of statistical inference. Davis and Mikosch (1998) have shown that for short-memory ARCH and GARCH models, the asymptotic properties of the sample autocorrelations are more severe, as there is no convergence in distribution. Now, for DDLRD, the behavior is somewhere in between the linear long memory and ARCH/GARCH cases, since for DDLRD the sample autocorrelations do converge in distribution for all $d$ with $0<d<1 / 2$, but the limiting distribution has infinite variance, and depends on $d$. Thus, the properties of parametric estimators of $d$ which use a fixed number of sample autocovariances will be strongly affected by the presence of DDLRD. 
Figure 3: QQ Plots of the Normalized Fourier Cosine Coefficients $A_{j} / f\left(\omega_{j}\right)^{\frac{1}{2}}$ for Parke process; $\mathrm{n}=10000, \mathrm{~d}=0.1$
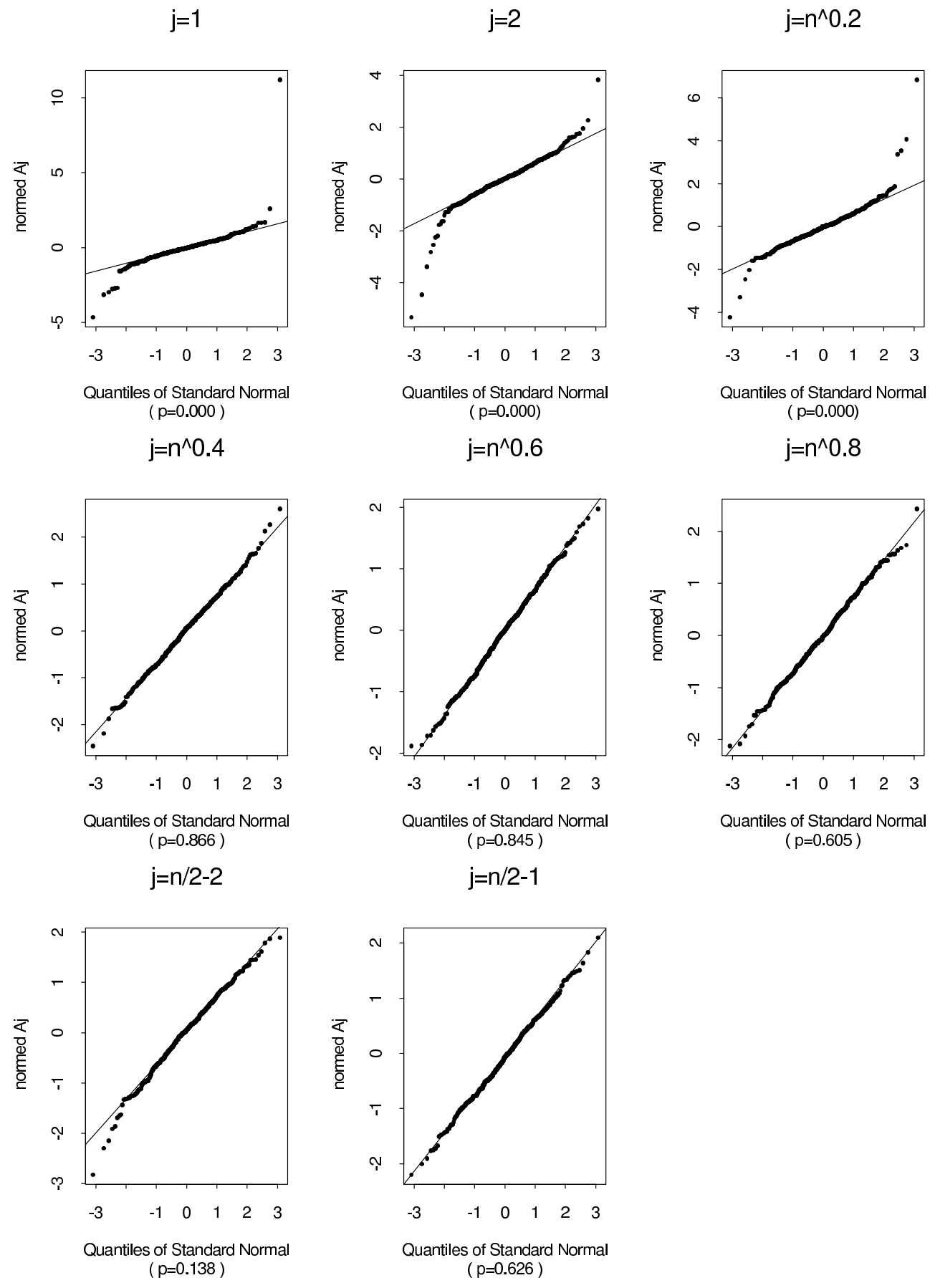
Figure 4: QQ Plots of the Normalized Fourier Cosine Coefficients $A_{j} / f\left(\omega_{j}\right)^{\frac{1}{2}}$ for Parke process; $\mathrm{n}=10000, \mathrm{~d}=0.4$
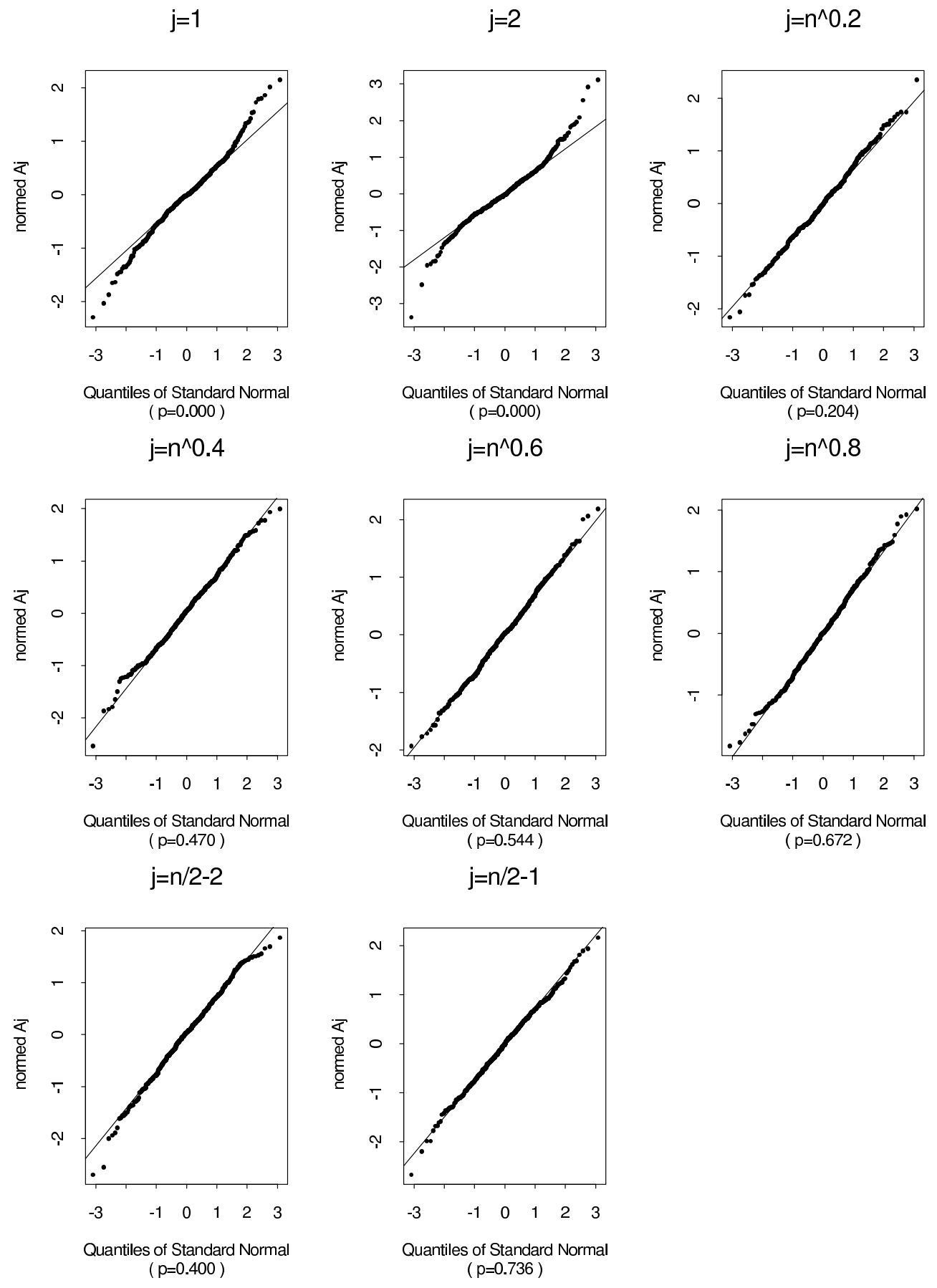
Figure 5: Scatterplots of Average Log Normalized Periodogram vs. $\quad \log \left|2 \sin \left(x_{j} / 2\right)\right|$; $\mathrm{j}=1,2, \ldots, 4999$. Horizontal line represents $-\gamma=-0.577216$.

Taqqu-Levy Process; d=0.1

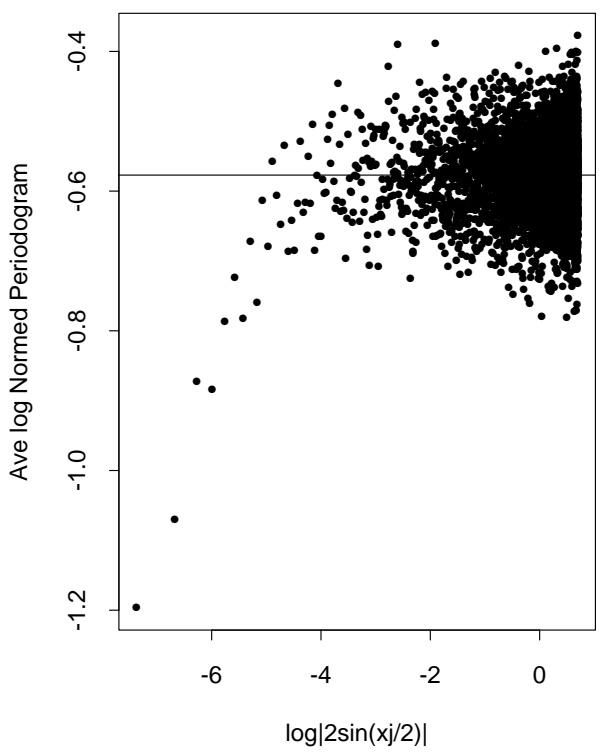

Parke Process; $\mathrm{d}=0.1$

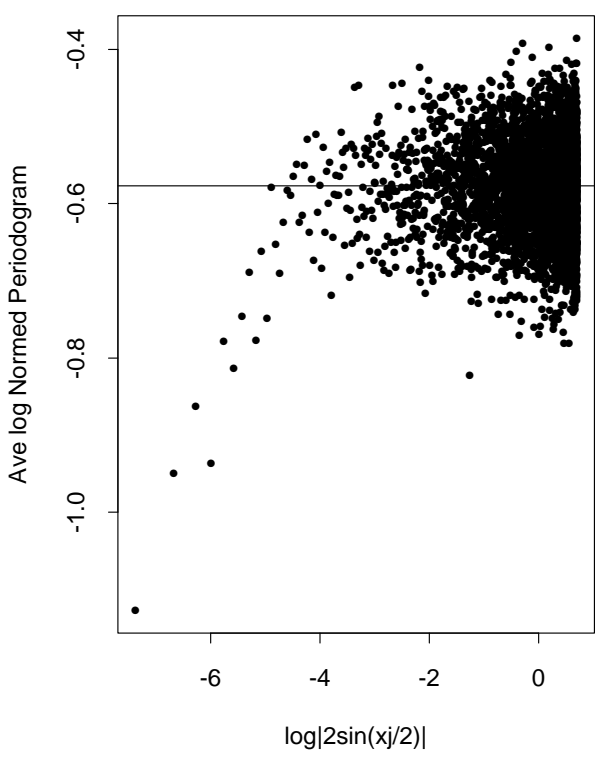

Taqqu-Levy Process; $d=0.4$

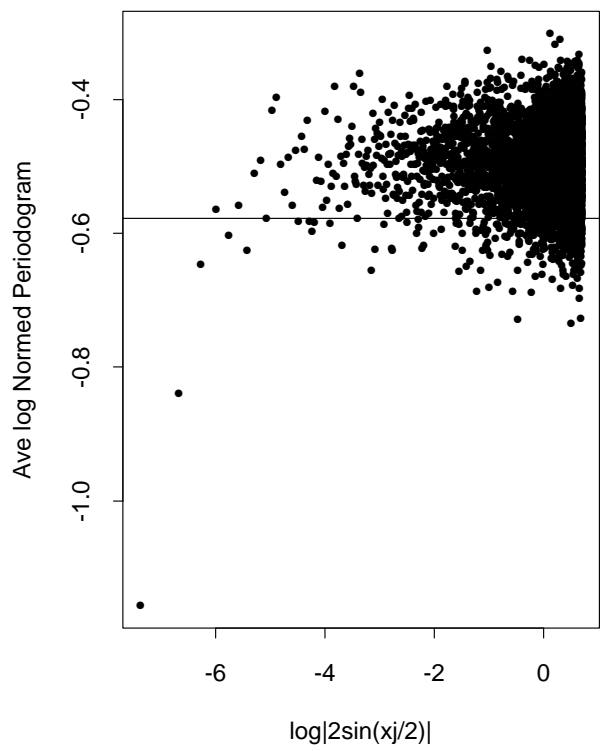

Parke Process; $d=0.4$

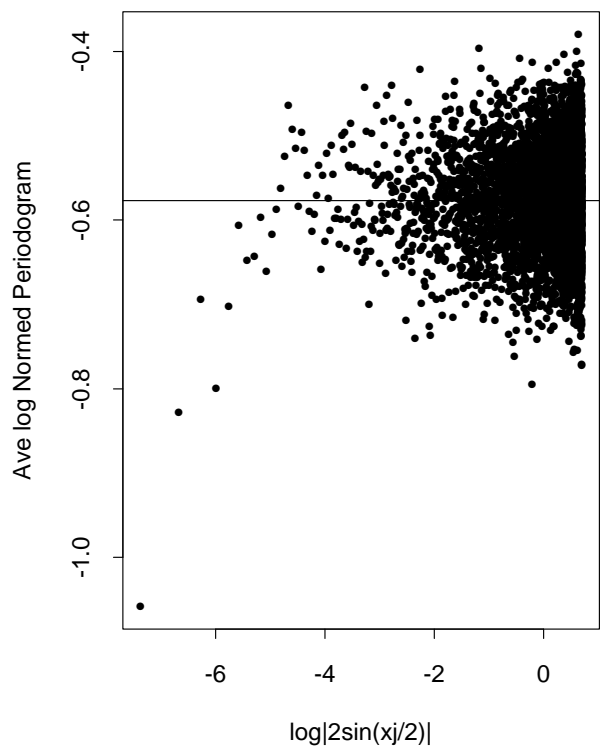


Figure 6: Scatterplots of Average Log Periodogram vs. $\log \left|2 \sin \left(x_{j} / 2\right)\right| ; j=1,2, \ldots, 4999$
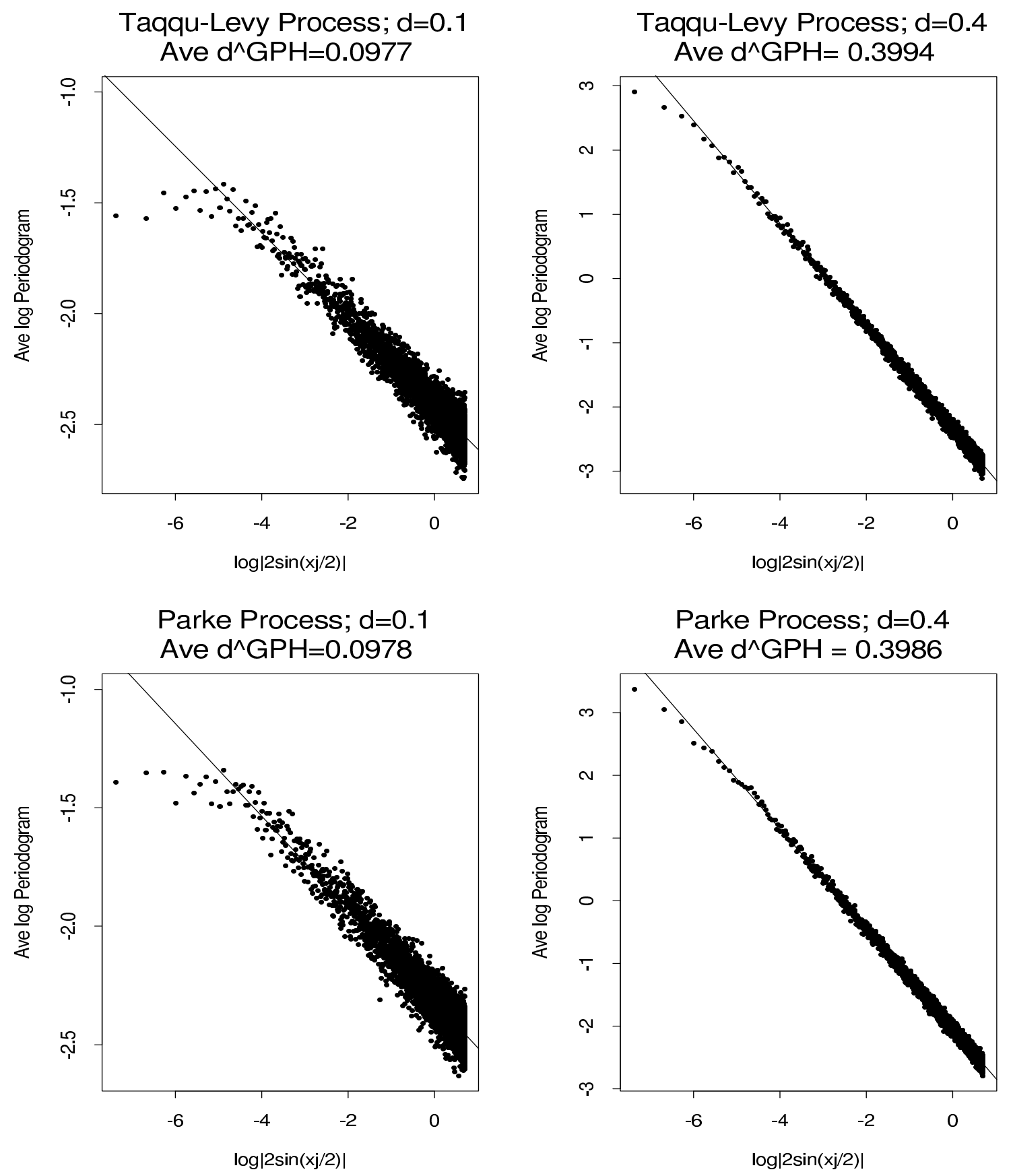
Figure 7: Normal QQ Plots of Sample Autocorrelations for Taqqu-Levy Process, $d=0.1$ lag 2

$\operatorname{lag} 20$
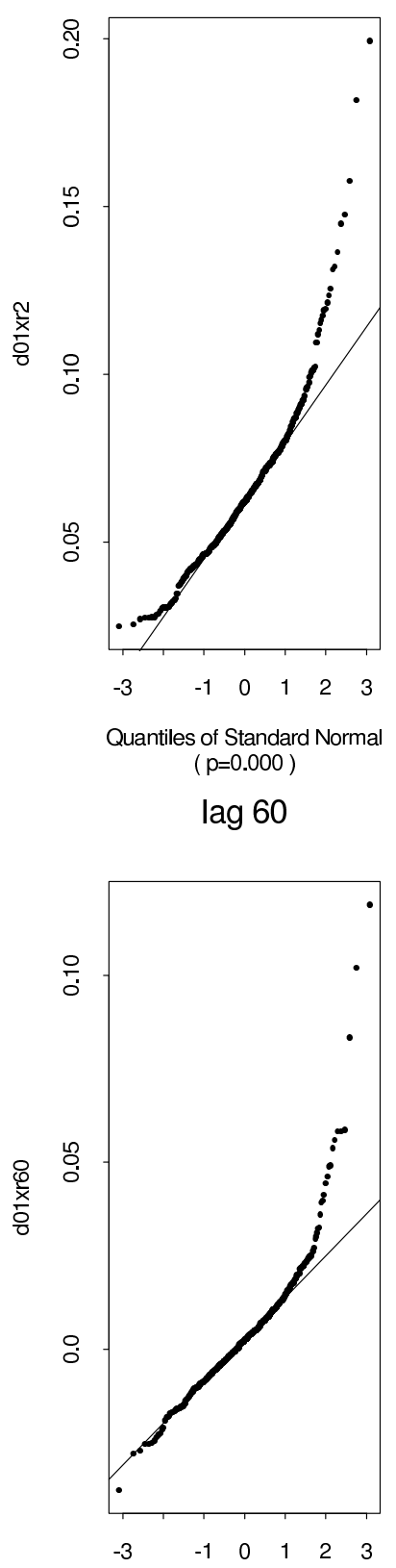

Quantiles of Standard Normal ( $p=0.000$ )
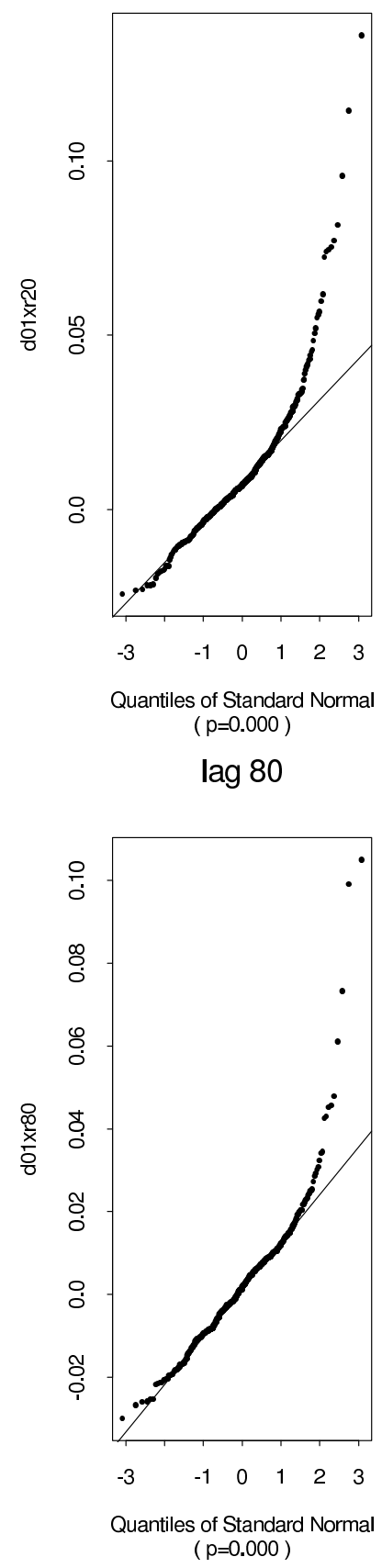

$\operatorname{lag} 40$
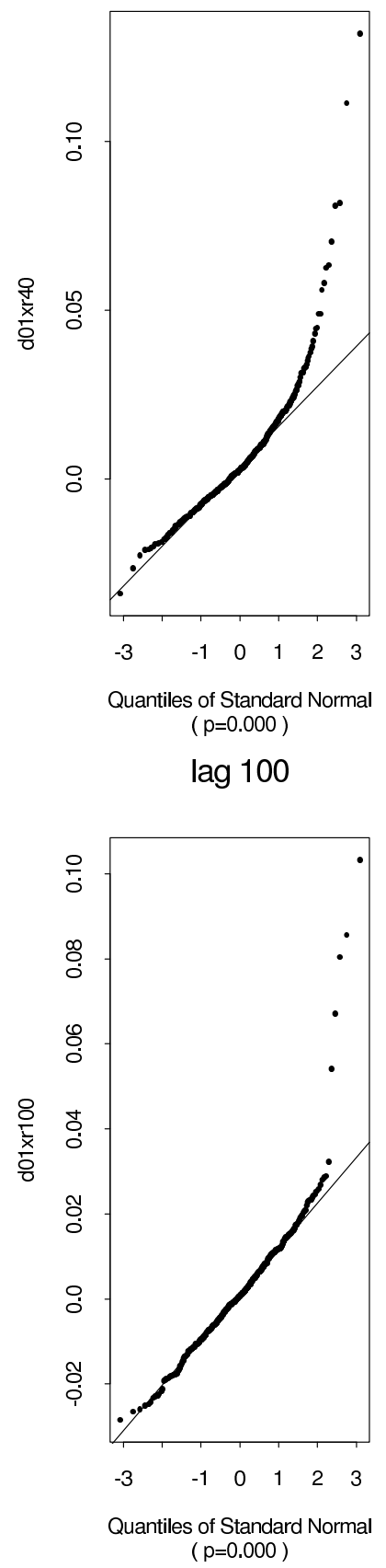
Table 1: Simulation variances for normalized DFT cosine coefficients at frequency $x_{j}$ for TaqquLevy Process, with normal-based 95\% Confidence Intervals. $\alpha=0.05$. Intervals marked with $*$ reject the null hypothesis, $\sigma^{2}=0.5$.

\begin{tabular}{|r|cc|cc|}
\hline$d$ & $x_{j}$ & Variance & Confidence Interval \\
\hline \hline 0.1 & $n^{0.2}$ & 0.54 & 0.48 & 0.62 \\
& $n^{0.4}$ & 0.54 & 0.47 & 0.61 \\
& $n^{0.6}$ & 0.49 & 0.44 & 0.56 \\
& $n^{0.8}$ & 0.53 & 0.47 & 0.60 \\
& $\frac{n}{2}-2$ & 0.51 & 0.45 & 0.58 \\
& $\frac{n}{2}-1$ & 0.50 & 0.44 & 0.56 \\
\hline \hline 0.4 & $n^{0.2}$ & 0.56 & 0.49 & 0.63 \\
& $n^{0.4}$ & 0.55 & 0.49 & 0.62 \\
& $n^{0.6}$ & 0.52 & 0.46 & 0.59 \\
& $n^{0.8}$ & 0.55 & 0.49 & 0.63 \\
& $\frac{n}{2}-2$ & 0.55 & 0.48 & 0.62 \\
& $\frac{n}{2}-1$ & 0.58 & 0.51 & $0.66^{*}$ \\
\hline
\end{tabular}

Table 2: Simulation variances for normalized DFT cosine coefficients at frequency $x_{j}$ for Parke Process, with normal-based $95 \%$ Confidence Intervals. $\alpha=0.05$. Intervals marked with $*$ reject the null hypothesis, $\sigma^{2}=0.5$.

\begin{tabular}{|c|cc|cc|}
\hline$d$ & $x_{j}$ & Variance & \multicolumn{2}{|c|}{ Confidence Interval } \\
\hline \hline 0.1 & $n^{0.2}$ & 0.66 & 0.58 & $0.75^{*}$ \\
& $n^{0.4}$ & 0.54 & 0.48 & 0.61 \\
& $n^{0.6}$ & 0.46 & 0.41 & 0.53 \\
& $n^{0.8}$ & 0.51 & 0.45 & 0.58 \\
& $\frac{n}{2}-2$ & 0.49 & 0.44 & 0.56 \\
& $\frac{n}{2}-1$ & 0.46 & 0.41 & 0.53 \\
\hline \hline 0.4 & $n^{0.2}$ & 0.47 & 0.42 & 0.54 \\
& $n^{0.4}$ & 0.49 & 0.44 & 0.56 \\
& $n^{0.6}$ & 0.46 & 0.41 & 0.53 \\
& $n^{0.8}$ & 0.47 & 0.42 & 0.53 \\
& $\frac{n}{2}-2$ & 0.54 & 0.48 & 0.62 \\
& $\frac{n}{2}-1$ & 0.52 & 0.46 & 0.59 \\
\hline
\end{tabular}




\section{APPENDIX}

\section{A Lemmas}

We present some lemmas in this section. Most of these are presumably known, but we were unable to find references for them under the conditions we needed for our main results. We therefore include proofs for the sake of completeness.

Lemma A.1. Let $\left(\zeta_{k}\right)_{k \in \mathbb{N}^{*}}$ be a martingale difference sequence such that $\sup _{k \geq 1} \mathbb{E}\left[\left|\zeta_{k}\right|^{p}\right]<\infty$ for all $p<\alpha$. Then, for any slowly varying function $h$,

$$
\sum_{k=1}^{M_{n}} \zeta_{k}-\sum_{k=1}^{[n / \mu]} \zeta_{k}=o_{P}\left(h(n) n^{1 / \alpha}\right) .
$$

Proof. To simplify the notation, without loss of generality, we can assume that $\mu=1$. For all $m$, denote $S_{m}=\sum_{k=1}^{m} \zeta_{k}$. By Theorem 2.5.15 in Embrechts et al. (1997), there exists a slowly varying function $\ell$ such that $\ell(n)^{-1} n^{-1 / \alpha}\left(M_{n}-n\right)$ converges in distribution to a stable law. Thus, for any sequence $\delta_{n}$ tending to infinity, we have:

$$
\lim _{n \rightarrow \infty} \mathbb{P}\left(\left|M_{n}-n\right| \geq \delta_{n} \ell(n) n^{1 / \alpha}\right)=0 .
$$

Let $\epsilon>0$ and $\delta_{n}$ be an arbitrary sequence tending to infinity. For any slowly varying function $h$, we can write:

$$
\begin{aligned}
& \mathbb{P}\left(\left|S_{M_{n}}-S_{n}\right| \geq \epsilon n^{1 / \alpha} h(n)\right) \\
& \leq \mathbb{P}\left(\left|M_{n}-n\right|>\delta_{n} n^{1 / \alpha} \ell(n)\right)+\mathbb{P}\left(\left|M_{n}-n\right| \leq \delta_{n} n^{1 / \alpha} \ell(n) ;\left|S_{M_{n}}-S_{n}\right| \geq \epsilon n^{1 / \alpha} h(n)\right) \\
& \quad \leq \mathbb{P}\left(\left|M_{n}-n\right|>\delta_{n} n^{1 / \alpha} \ell(n)\right)+\mathbb{P}\left(\max _{m:|m-n| \leq \delta_{n} n^{1 / \alpha} \ell(n)}\left|S_{n}-S_{m}\right| \geq \epsilon n^{1 / \alpha} h(n)\right) .
\end{aligned}
$$

Fix some $p \in(1, \alpha)$ and denote $C_{p}=\sup _{k \geq 1} \mathbb{E}\left[\left|\zeta_{k}\right|^{p}\right]<\infty$ by assumption. Denote By Kolmogorov's and Burkholder's inequalities (cf. Hall and Heyde (1980), Theorems 2.1 and 2.10), we obtain:

$$
\begin{aligned}
\mathbb{P}\left(\max _{m:|m-n| \leq n \delta_{n} n^{1 / \alpha} \ell(n)} \mid\right. & \left.S_{n}-S_{m} \mid \geq \epsilon n^{1 / \alpha} h(n)\right) \\
& \leq c \epsilon^{-1} n^{-1 / \alpha} h(n)^{-1} \mathbb{E}\left[\left|S_{n+\delta_{n} n^{1 / \alpha} \ell(n)}-S_{n-\delta_{n} n^{1 / \alpha} \ell(n)}\right|^{p}\right]^{1 / p} \\
& \leq c \epsilon^{-1} n^{-1 / \alpha} h(n)^{-1}\left(\sum_{k=n-\delta_{n} n^{1 / \alpha} \ell(n)}^{n+\delta_{n} n^{1 / \alpha} \ell(n)} \mathbb{E}\left[\left|\zeta_{k}\right|^{p}\right]\right)^{1 / p} \\
& \leq c C_{p} \epsilon^{-1} n^{-1 / \alpha} h(n)^{-1}\left(\delta_{n} n^{1 / \alpha} \ell(n)\right)^{1 / p} .
\end{aligned}
$$

Since $p>1$, this last term is $o(1)$ is the sequence $\delta_{n}$ converges to infinity slowly enough. 
Lemma A.2. Let $\left(\zeta_{n, k}\right)_{1 \leq k \leq n}$ be uniformly bounded random variables. Let $\left(T_{k}\right)_{k \geq 1}$ be i.i.d. random variables that satisfy (3.4) for some $\alpha \in(1,2)$ and such that for all $n \geq 1$ and all $k \leq n, T_{k}$ is independent of $\left\{\zeta_{n, j}, 1 \leq j<k\right\}$. Let $W_{k}$ be i.i.d. random variables with zero mean and finite variance, independent of $\zeta_{n, k}, 1 \leq k \leq n$ and $T_{k}, 1 \leq k \leq n$. Let $H$ be a bounded continuous function such that for all $u \in \mathbb{R}$ and $v \in(0,1)$ :

$$
|H(u, v)-u| \leq C|u|\left\{u^{2} v^{2} \wedge 1+v^{2}\right\} .
$$

If $m \leq$ cn and $j \leq n^{\rho}$ for some $\rho \in(0,1 / \alpha)$, then $\sum_{k=1}^{m} \zeta_{n, k} W_{k}\left\{H\left(T_{k}, x_{n, j}\right)-T_{k}\right\}=o_{P}\left(n^{1 / \alpha} \ell(n)\right)$ for any slowly varying function $\ell$.

Proof. Define $\xi_{n}=\sum_{k=1}^{n} \zeta_{n, k}\left\{H\left(j T_{k} / n\right)-j T_{k} / n\right\} W_{k}$ and let $\mathbb{E}_{T, \zeta}$ denote the conditional expectation with respect to all the variables $\zeta_{n, k}$ and $T_{k}$. Since the variables $\zeta_{n, k}$ are uniformly bounded, and since, for $p \in[1, \alpha)$, the function $x \rightarrow x^{p / 2}$ is concave, we obtain:

$$
\mathbb{E}_{T, \zeta}\left[\left|\xi_{n}\right|^{p}\right] \leq C\left\{\sum_{k=1}^{n}\left\{H\left(T_{k}, x_{n, j}\right)-T_{k}\right\}^{2}\right\}^{p / 2} \leq C \sum_{k=1}^{n}\left|H\left(T_{k}, x_{n, j}\right)-T_{k}\right|^{p} .
$$

Hence, taking expectations on both sides and applying (A.2), we obtain:

$$
\begin{aligned}
\mathbb{E}\left[\left|\xi_{n}\right|^{p}\right] & \leq C \sum_{k=1}^{n} \mathbb{E}\left[\left|H\left(T_{k}, x_{n, j}\right)-T_{k}\right|^{p}\right] \\
& \leq C n \mathbb{E}\left[\left|T_{1}\right|^{p}\left(\left|j T_{1} / n\right|^{2} \wedge 1\right)^{p}\right]+C n(j / n)^{2 p} \leq C n L(n)\left\{(j / n)^{\alpha-p}+(j / n)^{2 p}\right\} .
\end{aligned}
$$

Thus, $\xi_{n}=O_{P}\left(\{n L(n)\}^{1 / p}\left\{j^{\alpha / p-1}+(j / n)^{2}\right\}\right)$. If $\rho<1-1 / \alpha$, then $p$ can be chosen such that $\{n L(n)\}^{1 / p}\left\{j^{\alpha / p-1}+(j / n)^{2}\right\}=o\left(n^{1 / \alpha} \ell(n)\right)$. Hence $\xi_{n}=o_{P}\left(n^{1 / \alpha} \ell(n)\right)$ for any slowly varying function $\ell$.

Lemma A.3. Let $\zeta_{k}$ be a sequence of i.i.d. rv such that for all $p \in(1, \alpha), \mathbb{E}\left[\left|\zeta_{k}\right|^{p}\right]<\infty, \mathbb{E}\left[\zeta_{k}\right]=0$ and $\zeta_{k}$ is independent of $S_{0}, T_{1}, \ldots, T_{k-1}$. Let $K$ be a bounded continuously differentiable function on $\mathbb{R}$, with bounded derivative. Define $U_{m, n, j}=\sum_{k=1}^{m} K\left(S_{k-1} x_{n, j}\right) \zeta_{k}$ and $V_{m, n, j}=\sum_{k=1}^{m} K((k-$ 1) $\left.\mu x_{n, j}\right) \zeta_{k}$. If $m \leq c n$ and $j \leq n^{\rho}$ for some $\rho \in\left(0,1-1 / \rho\right.$, then $U_{m, n, j}-V_{m, n, j}=o_{P}\left(n^{1 / \alpha} \ell(n)\right)$ for any slowly varying function $\ell$.

Proof. Denote $R_{k}=T_{1}+\cdots+T_{k}-k \mu$. Since $K$ is differentiable, we can write:

$$
\begin{aligned}
U_{m, n}-V_{m, n} & =\sum_{k=1}^{m} K^{\prime}\left((k-1) \mu x_{n, j}+\varsigma_{k}\left(S_{k-1}-(k-1) \mu x_{n, j}\right)\left\{S_{k-1}-(k-1) \mu\right\} x_{n, j} \zeta_{k}\right. \\
& =x_{n, j} \sum_{k=1}^{n} \rho_{n, k} R_{k-1} \zeta_{k}+S_{0} x_{n, j} \sum_{k=1}^{n} \rho_{n, k} \zeta_{k},
\end{aligned}
$$

where $\rho_{n, k}=K^{\prime}\left(\left\{(k-1) \mu+\varsigma_{k}\left(S_{k-1}-(k-1) \mu\right\} / n\right)\right.$. Since $\mathbb{E}\left[\left|\zeta_{k}\right|\right]<\infty$ and $K^{\prime}$ is bounded, the last term above is trivially $O_{P}(1)$. By assumption, $\left\{\sum_{j=1}^{k} \rho_{n, k} R_{k-1} \zeta_{k}, 1 \leq k \leq n\right\}$ is a martingale 
with finite $p$-th moment for $p<\alpha$. Hence by the Burkholder inequality for martingales, we have, for $p<\alpha, \mathbb{E}\left[\left|R_{k}\right|^{p}\right]=O(k)$ and

$$
\mathbb{E}\left[\left|\sum_{k=1}^{n} \rho_{n, k} R_{k-1} \zeta_{k}\right|^{p}\right] \leq C \sum_{k=1}^{n} \mathbb{E}\left[\left|R_{k-1}\right|^{p}\right]=O\left(n^{2}\right) .
$$

Thus $x_{n, j} \sum_{k=1}^{n} \rho_{n, k} R_{k-1} \zeta_{k}=O_{P}\left(j n^{2 / p-1}\right)$. If $\rho<1-1 / \alpha$, then $p$ can be chosen so that $j n^{2 / p-1}=o\left(n^{1 / \alpha} \ell(n)\right)$ for any slowly varying function $\ell$.

Lemma A.4. Let $H$ be a bounded continuously differentiable function on $\mathbb{R}$ such that $H(x)=$ $O\left(x^{2}\right)$ in a neighborhood of 0 . If $T_{1}$ satisfies (3.4), then

$$
\lim _{t \rightarrow \infty} t^{\alpha} L^{-1}(t) \mathbb{E}\left[H\left(T_{1} / t\right)\right]=\alpha \int_{0}^{\infty} H(s) s^{-\alpha-1} d s .
$$

Proof. Assume first that $H$ has a compact support in $(0, \infty)$ and is continuously differentiable. Then:

$$
\begin{aligned}
\mathbb{E}\left[H\left(T_{1} / t\right)\right] & =\sum_{k=1}^{\infty} H(k / t) \mathbb{P}(T=k)=\sum_{k=1}^{\infty} H(k / t)\{\mathbb{P}(T \geq k)-\mathbb{P}(T \geq k-1)\} \\
& \left.=\sum_{k=1}^{\infty} \mathbb{P}(T \geq k)\{H(k / t)-H((k-1) / t)\}=\int_{0}^{\infty} \mathbb{P}(T>\lfloor s\rfloor]+1\right) H^{\prime}(s / t) d s / t \\
& =\int_{0}^{\infty}(\lfloor s\rfloor+1)^{-\alpha} L(\lfloor s\rfloor+1) H^{\prime}(s / t) d s / t=\int_{0}^{\infty}(\lfloor t x\rfloor+1)^{-\alpha} L(\lfloor t x\rfloor+1) H^{\prime}(x) d x,
\end{aligned}
$$

Since $L$ is slowly varying, by Karamata's Theorem, we know that $\lim _{t \rightarrow \infty} L(t)^{-1} L(\lfloor t x\rfloor+1)=1$, uniformly with respect to $x$ in compact sets of $(0, \infty)$. Thus, since we have assumed that $H$ has compact support in $(0, \infty)$, we obtain

$$
\lim _{t \rightarrow \infty} t^{\alpha} L^{-1}(t) \mathbb{E}\left[H\left(T_{1} / t\right)\right]=\int_{0}^{\infty} x^{-\alpha} H^{\prime}(x) d x=\alpha \int_{0}^{\infty} x^{-\alpha-1} H(x) d x .
$$

To conclude, it is sufficient to prove that

$$
\lim _{A \rightarrow \infty} \limsup _{t \rightarrow \infty} t^{\alpha} L^{-1}(t) \mathbb{E}\left[H(T / t) \mathbf{1}_{\{T>A t \text { or } T<t / A\}}\right]=0 .
$$

This tightness property allows then to truncate the function $H$ and apply the first part of the proof. For any $A>0$ and $t$ large enough, applying the assumption on the behaviour of the function $H$ at zero, we have:

$$
\mathbb{E}\left[H(T / t) \mathbf{1}_{\{T<t / A\}}\right]=\sum_{k=1}^{t / A} H(k / t) \mathbb{P}(T=k) \leq C t^{-2} \sum_{k=1}^{t / A} k^{2} \mathbb{P}(T=k) .
$$


Applying summation by parts and Karamata's theorem, we obtain:

$$
\sum_{k=1}^{t / A} k^{2} \mathbb{P}(T=k)=1+\sum_{k=1}^{t / A} \mathbb{P}(T \geq n)\left\{k^{2}-(k-1)^{2}\right\} \leq C A^{\alpha-2} L(A t) .
$$

Thus, there exists a constant $C$ such that:

$$
\limsup _{t \rightarrow \infty} t^{\alpha} L^{-1}(t) \mathbb{E}\left[H(T / t) \mathbf{1}_{\{T<t / A\}}\right] \leq C A^{\alpha-2} \lim _{t \rightarrow \infty} L(A t) / L(t)=C A^{\alpha-2} .
$$

Similarly, we can show that

$$
\limsup _{t \rightarrow \infty} t^{\alpha} L^{-1}(t) \mathbb{E}\left[H(T / t) \mathbf{1}_{\{T>A t\}}\right] \leq C A^{-\alpha} \leq C A^{\alpha-2}
$$

This proves (A.3) and concludes the proof of Lemma A.4.

Lemma A.5. Let $j=j(n)$ be a sequence of integers such that $n^{\beta} \leq j \leq n^{\rho}$ for $0<\beta \leq \rho<1$. Then

$$
\begin{array}{r}
\mathbb{P}-\lim _{n \rightarrow \infty} n^{-1} \sum_{k=1}^{n} \cos \left(x_{j} S_{k}\right)=\mathbb{P}-\lim _{n \rightarrow \infty} n^{-1} \sum_{k=1}^{n} \sin \left(x_{j} S_{k}\right)=0, \\
\mathbb{P}-\lim _{n \rightarrow \infty} n^{-1} \sum_{k=1}^{n} \cos ^{2}\left(x_{j} S_{k}\right)=\mathbb{P}-\lim _{n \rightarrow \infty} n^{-1} \sum_{k=1}^{n} \sin ^{2}\left(x_{j} S_{k}\right)=1 / 2 .
\end{array}
$$

where $\mathbb{P}-\lim$ denotes convergence in probability.

Note that (A.5) follows from (A.4) by the relation $\cos ^{2}(u)=(1+\cos (2 u)) / 2$ and by replacing $j$ by $2 j$.

To prove Lemma A.5, we use the following theorem, which adapts Theorem 2 in Yong (1971).

Theorem A.1. Let $T$ be a non negative integer valued random variable in the domain of attraction of an $\alpha$-stable law with $\alpha \in(1,2)$, such that $\mathbb{P}(T \geq k)=k^{-\alpha} L(k)$, where $L$ is slowly varying at infinity. Let $\phi$ be the characteristic function of $T$. Then, for $z>0, \phi(z)=1-z^{\alpha} \ell_{1}(z)+\mathrm{i} \ell_{2}(z) z$ where $\ell_{1}$ and $\ell_{2}$ are slowly varying at zero, positive in a neighborhood of zero and satisfy, for some finite nonzero constant $C(\alpha)$,

$$
\lim _{x \rightarrow 0} \ell_{1}(x) / L(1 / x)=C(\alpha) \text { and } \lim _{x \rightarrow 0} \ell_{2}(x)=\mathbb{E}[T]>0 .
$$

We will use Theorem A.1 through the following bound for the modulus of the characteristic function of $T$ :

$$
|\phi(z)|^{2} \leq 1-2 \ell(z) z^{\alpha}
$$

where $\ell(z)=\ell_{1}(z)-\frac{1}{2} \ell_{1}^{2} z^{\alpha}-\frac{1}{2} \ell_{2}^{2}(z) z^{2-\alpha}$ is slowly varying and positive in a neighborhood of zero. 
Proof of LemmaA.5. We prove that the convergence holds in $L^{2}$. Write

$$
\begin{aligned}
\mathbb{E}\left[\left\{\frac{1}{n} \sum_{k=1}^{n} \cos \left(x_{j} S_{k}\right)\right\}^{2}\right] & =\frac{1}{n^{2}} \sum_{k=1}^{n} \mathbb{E}\left[\cos ^{2}\left(x_{j} S_{k}\right)\right]+\frac{2}{n^{2}} \sum_{k=2}^{n} \sum_{\ell=1}^{k-1} \mathbb{E}\left[\cos \left(x_{j} S_{\ell}\right) \cos \left(x_{j} S_{k}\right)\right] \\
& =O\left(n^{-1}\right)+\frac{1}{n^{2}} \sum_{k=2}^{n} \sum_{\ell=1}^{k-1} \mathbb{E}\left[\cos \left(x_{j}\left(S_{\ell}+S_{k}\right)\right)+\cos \left(x_{j}\left(S_{k}-S_{\ell}\right)\right)\right], \\
\mathbb{E}\left[\left\{\frac{1}{n} \sum_{k=1}^{n} \sin \left(x_{j} S_{k}\right)\right\}^{2}\right] & =O\left(n^{-1}\right)+\frac{1}{n^{2}} \sum_{k=2}^{n} \sum_{\ell=1}^{k-1} \mathbb{E}\left[\cos \left(x_{j}\left(S_{k}-S_{\ell}\right)\right)-\cos \left(x_{j}\left(S_{k}+S_{\ell}\right)\right)\right] .
\end{aligned}
$$

Thus we have to show that

$$
\begin{aligned}
& \lim _{n \rightarrow \infty} \frac{1}{n^{2}} \sum_{k=2}^{n} \sum_{\ell=1}^{k-1} \mathbb{E}\left[\cos \left(x_{j}\left(S_{k}-S_{\ell}\right)\right)\right]=0, \\
& \lim _{n \rightarrow \infty} \frac{1}{n^{2}} \sum_{k=2}^{n} \sum_{\ell=1}^{k-1} \mathbb{E}\left[\cos \left(x_{j}\left(S_{\ell}+S_{k}\right)\right)\right]=0 .
\end{aligned}
$$

Proof of A.7). Applying (A.6), for large enough $n$, we have

$$
\left|\frac{1}{n^{2}} \sum_{k=2}^{n} \sum_{k^{\prime}=1}^{k-1} \mathbb{E}\left[\cos \left(x_{j}\left(S_{k}-S_{k^{\prime}}\right)\right)\right]\right| \leq \frac{1}{n^{2}} \sum_{k=2}^{n} \sum_{k^{\prime}=1}^{k-1}\left|\phi\left(x_{j}\right)\right|^{k-k^{\prime}} \leq \frac{1}{n} \sum_{k=1}^{n-1}\left\{1-2 \ell\left(x_{j}\right) x_{j}^{\alpha}\right\}^{k / 2} .
$$

For $z \in(0,1)$ and any real number $t \geq 1,(1-z)=\mathrm{e}^{t \log (1-z)} \leq \mathrm{e}^{-t z}$ and

$$
\frac{1}{n} \sum_{k=1}^{n}(1-z)^{k / 2} \leq \frac{1}{n} \sum_{k=1}^{n} \mathrm{e}^{-k z / 2}=\frac{1-\mathrm{e}^{-n z / 2}}{n\left(\mathrm{e}^{z / 2}-1\right)} \leq \frac{1-e^{-n z / 2}}{n z / 2} .
$$

Hence:

$$
\left|\frac{1}{n^{2}} \sum_{k=2}^{n} \sum_{k^{\prime}=1}^{k-1} \mathbb{E}\left[\cos \left(x_{j}\left(S_{k}-S_{k^{\prime}}\right)\right)\right]\right| \leq \frac{1}{n} \sum_{k=1}^{n-1}\left\{1-2 \ell\left(x_{j}\right) x_{j}^{\alpha}\right\}^{k / 2} \leq \frac{1-\mathrm{e}^{-\ell\left(x_{j}\right) n x_{j}^{\alpha}}}{n \ell\left(x_{j}\right) x_{j}^{\alpha}} .
$$

Under the assumption on the sequence $j, \lim _{n \rightarrow \infty} n \ell\left(x_{j}\right) x_{j}^{\alpha}=\infty$. Thus the limit of the last term in (A.9) is 0 . This concludes the proof of (A.7).

Proof of (A.8). Since $S_{k}+S_{k^{\prime}}=2 S_{0}+2\left(T_{1}+\cdots+T_{k^{\prime}}\right)+T_{k^{\prime}+1}+\cdots+T_{k}$, and denoting $\phi_{0}$ the characteristic function of $S_{0}$, we have

$$
\left|\frac{1}{n^{2}} \sum_{k=2}^{n} \sum_{k^{\prime}=1}^{k-1} \mathbb{E}\left[\cos \left(x_{j}\left(S_{k}+S_{k^{\prime}}\right)\right)\right]\right| \leq \frac{1}{n^{2}} \sum_{k=2}^{n} \sum_{k^{\prime}=1}^{k-1}\left|\phi_{0}\left(2 x_{j}\right)\right|\left|\phi\left(2 x_{j}\right)\right|^{k^{\prime}}\left|\phi\left(x_{j}\right)\right|^{k-k^{\prime}} .
$$


Applying (A.6), for large enough $n$, we obtain:

$$
\left|\frac{1}{n^{2}} \sum_{k=2}^{n} \sum_{k^{\prime}=1}^{k-1} \mathbb{E}\left[\cos \left(x_{j}\left(S_{k}+S_{k^{\prime}}\right)\right)\right]\right| \leq \frac{1}{n^{2}} \sum_{k=2}^{n} \sum_{k^{\prime}=1}^{k-1}\left\{1-2 \ell\left(2 x_{j}\right)\left(2 x_{j}\right)^{\alpha}\right\}^{k^{\prime}}\left\{1-2 \ell\left(x_{j}\right) x_{j}^{\alpha}\right\}^{k-k^{\prime}} .
$$

Since for any slowly varying function $L$ and any $\alpha>0$ the function $z^{\alpha} L(z)$ is ultimately non decreasing, we obtain, for $n$ large enough:

$$
\mid \frac{1}{n^{2}} \sum_{k=2}^{n} \sum_{k^{\prime}=1}^{k-1} \mathbb{E}\left[\cos \left(x_{j}\left(S_{k}+S_{k^{\prime}}\right)\right] \mid \leq \frac{1}{n} \sum_{k=2}^{n}\left\{1-2 \ell\left(x_{j}\right) x_{j}^{\alpha}\right\}^{k / 2} .\right.
$$

The same line of reasoning as previously concludes the proof of A.8 and of Lemma A.5.

\section{B Proof of the main results}

Proof of Proposition 3.2. A necessary and sufficient condition for this process to be well defined is that almost surely, for all $t \in \mathbb{Z}$,

$$
\inf \left\{s \in \mathbb{Z}, s+n_{s} \geq t\right\}>-\infty \text {. }
$$

Since the random variables $n_{s}$ are i.i.d. with the same distribution as $N$, by Borel-Cantelli's Lemma this condition is equivalent to

$$
\sum_{s \leq t} \mathbb{P}\left(n_{s} \geq t-s\right)=\sum_{k=0}^{\infty} \mathbb{P}(N \geq k)<\infty
$$

Hence the necessary and sufficient condition for Parke's error duration process to be well defined is $\mathbb{E}[N]<\infty$. The expression of the autocovariance function is proved in Parke (1999) Proposition 1.

Proof of Proposition 3.3 in the case of Parke's process. For any real numbers $x, y$, denote $x_{+}=$ $\max (x, 0), x_{-}=\max (-x, 0), x \vee y=\max (x, y)$ and $x \wedge y=\min (x, y)$. Recall that $g_{s, t}=1$ if $s \leq t \leq s+n_{s}$ and 0 otherwise. Hence, we can write:

$$
\begin{aligned}
\sum_{k=1}^{n} X_{k} & =\sum_{k=1}^{n} \sum_{s \leq k} g_{s, k} \epsilon_{s}=\sum_{s \leq n} \sum_{k=1 \vee s}^{\left(s+n_{s}\right)_{+} \wedge n} g_{s, k} \epsilon_{s} \\
& =\sum_{s \leq 0}\left\{\left(s+n_{s}\right)_{+} \wedge n\right\} \epsilon_{s}+\sum_{s=1}^{n}\left\{\left(s+n_{s}\right) \wedge n-s+1\right\} \epsilon_{s}=U_{n}+V_{n}
\end{aligned}
$$

Since $\sum_{s \leq 0} \mathbb{P}\left(s+n_{s}>0\right)=\sum_{k \geq 0} \mathbb{P}(N>k)=\mathbb{E}[N]<\infty$, the number of terms in the sum $U=\sum_{s \leq 0}\left(s+n_{s}\right)_{+} \epsilon_{s}$ is almost surely finite. Hence $U_{n}$ converges almost surely to $U$ and 
$U_{n}=O_{P}(1)$. We now split $V_{n}$ into three terms: $V_{n}=V_{1, n}-V_{2, n}+V_{3, n}$, with

$$
\begin{gathered}
V_{1, n}=\sum_{s=1}^{n}\left(n_{s}+1\right) \epsilon_{s}, \quad V_{2, n}=\sum_{s=1}^{n}\left(n_{s}+1\right) \mathbf{1}_{\left\{s+n_{s}>n\right\}} \epsilon_{s}, \\
\quad \text { and } \quad V_{3, n}=\sum_{s=1}^{n}(n-s+1) \mathbf{1}_{\left\{s+n_{s}>n\right\}} \epsilon_{s} .
\end{gathered}
$$

Since the sequences $\left(n_{s}\right)$ and $\left(\epsilon_{s}\right)$ are i.i.d. and independent of each other, $V_{3, n}$ has the same distribution as $W_{n}=\sum_{k=1}^{n} k \mathbf{1}_{\left\{n_{k} \geq k\right\}} \epsilon_{k}$. Since $\sum_{k=1}^{\infty} \mathbb{P}\left(n_{k} \geq k\right)<\infty$, by Borel-Cantelli's Lemma, almost surely there exists an integer $K$ such that for all $k>K, n_{k}<k$. Hence $W_{n}$ converges almost surely to $\sum_{k=1}^{\infty} k \mathbf{1}_{\left\{n_{k} \geq k\right\}} \epsilon_{k}$, which is almost surely a finite sum. This implies that $V_{3, n}=O_{P}(1)$.

Similarly, $V_{2, n}$ has the same distribution as $\sum_{k=1}^{n} n_{k} \mathbf{1}_{\left\{n_{k} \geq k\right\}} \epsilon_{k}$, which converges almost surely to the almost surely finite sum $\sum_{k=1}^{\infty} n_{k} \mathbf{1}_{\left\{n_{k} \geq k\right\}} \epsilon_{k}$. Hence $V_{2, n}=O_{P}(1)$.

Under assumption (3.4),$N$ is in the domain of attraction of an $\alpha$-stable law. Since $\mathbb{E}\left[\epsilon_{0}^{2}\right]<\infty$, by Breiman's (1965) theorem, $\left(n_{s}+1\right) \epsilon_{s}$ is an i.i.d. sequence in the domain of attraction of an $\alpha$-stable law. Thus we obtain that $n^{-1 / \alpha} \ell(n)^{-1} V_{1, n}$ converges weakly to the stable distribution with characteristic function given by (3.5) (cf. for instance Embrechts et al. (1997), Proposition 2.2.13). The convergence of finite dimensional distribution is obtained similarly.

Sketch of Proof of Theorem 3.1. Neglecting the first and last renewal periods, write

$$
\begin{aligned}
& \ell(n)^{-1} n^{1-1 / \alpha}\left\{\hat{F}_{n}(x)-F_{W}(x)\right\}=\ell(n)^{-1} n^{-1 / \alpha} \sum_{k=1}^{M_{n}} \mathbf{1}_{\left\{W_{k} \leq x\right\}}\left(T_{k}-\mu\right) \\
& \quad+\mu \ell(n)^{-1} n^{-1 / \alpha} \sum_{k=1}^{M_{n}}\left\{\mathbf{1}_{\left\{W_{k} \leq x\right\}}-F_{W}(x)\right\}+\left(\mu \ell(n)^{-1} n^{-1 / \alpha} M_{n}-1\right) F_{W}(x)+o_{P}(1) .
\end{aligned}
$$

By Lemma A.1, the finite dimensional distributions of $\ell(n) n^{-1 / \alpha} \sum_{k=1}^{M_{n}} \mathbf{1}_{\left\{W_{k} \leq x\right\}}\left(T_{k}-\mu\right)$ are asymptotically equivalent to those of $\ell(n) n^{-1 / \alpha} \sum_{k=1}^{n / \mu} \mathbf{1}_{\left\{W_{k} \leq x\right\}}\left(T_{k}-\mu\right)$ which converge to those of $\Lambda_{\alpha}(F(x))$. Since the variables $W_{k}$ are independent of $M_{n}$, we have, by the renewal theorem,

$$
\mathbb{E}\left[\left(\sum_{k=1}^{M_{n}}\left\{\mathbf{1}_{\left\{W_{k} \leq x\right\}}-F_{W}(x)\right\}\right)^{2}\right]=F_{W}(x)\left\{1-F_{W}(x)\right\} \mathbb{E}\left[M_{n}\right]=O(n) .
$$

Thus, $\sum_{k=1}^{M_{n}}\left\{\mathbf{1}_{\left\{W_{k} \leq x\right\}}-F_{W}(x)\right\}=o_{P}\left(\ell(n) n^{1 / \alpha}\right)$. 
Proof of Proposition 4.1 in the case of Parke's process.

$$
\begin{aligned}
D_{n, j} & :=\sum_{t=1}^{n} X_{t} \mathrm{e}^{\mathrm{i} t x_{j}}=\sum_{t=1}^{n} \sum_{s \leq t} g_{s, t} \mathrm{e}^{\mathrm{i} t x_{j}} \epsilon_{s} \\
& =\sum_{s \leq 0} \sum_{t=1}^{\left(s+n_{s}\right)_{+} \wedge n} \mathrm{e}^{\mathrm{i} t x_{j}} \epsilon_{s}+\sum_{s=1}^{n} \sum_{t=s}^{\left(s+n_{s}\right) \wedge n} \mathrm{e}^{\mathrm{i} t x_{j}} \epsilon_{s}=: U_{n, j}+V_{n, j} .
\end{aligned}
$$

As in the proof of Proposition 3.3, the sum defining $U_{n, j}$ is almost surely finite. If $j / n \rightarrow 0$, then $U_{n, j}$ converges almost surely to the random variable $U=\sum_{s \leq 0}\left(s+n_{s}\right)_{+} \epsilon_{s}$. Split now $V_{n, j}$ into three terms: $V_{n, j}=W_{n, j}-R_{n, j}+T_{n, j}$, with

$$
\begin{gathered}
W_{n, j}=\sum_{s=1}^{n}\left(\sum_{t=s}^{\left(s+n_{s}\right)} \mathrm{e}^{\mathrm{i} t x_{j}}\right) \epsilon_{s}, \\
R_{n, j}=\sum_{s=1}^{n} \sum_{t=s}^{\left(s+n_{s}\right)} \mathrm{e}^{\mathrm{i} t x_{j}} \mathbf{1}_{\left\{s+n_{s}>n\right\}} \epsilon_{s}, \\
T_{n, j}=\sum_{s=1}^{n} \sum_{t=s}^{n} \mathrm{e}^{\mathrm{i} t x_{j}} \mathbf{1}_{\left\{s+n_{s}>n\right\}} \epsilon_{s} .
\end{gathered}
$$

Consider first $R_{n}$. Since the sequences $\left(n_{s}\right)$ and $\left(\epsilon_{s}\right)$ are i.i.d. and independent of each other, we have:

$$
R_{n, j}=\sum_{s=1}^{n} \mathrm{e}^{\mathrm{i} s x_{j}} \frac{1-\mathrm{e}^{\mathrm{i}\left(n_{s}+1\right) x_{j}}}{1-\mathrm{e}^{\mathrm{i} x_{j}}} \mathbf{1}_{\left\{s+n_{s}>n\right\}} \epsilon_{s} \stackrel{(d)}{=} \sum_{k=1}^{n} \mathrm{e}^{-\mathrm{i}(k-1) x_{j}} \frac{1-\mathrm{e}^{\mathrm{i}\left(n_{k}+1\right) x_{j}}}{1-\mathrm{e}^{\mathrm{i} x_{j}}} \mathbf{1}_{\left\{n_{k} \geq k\right\}} \epsilon_{k},
$$

where $\stackrel{(d)}{=}$ denotes equality of laws. Since almost surely there is only a finite number of indices $k$ such that $n_{k} \geq k$, if $j / n \rightarrow 0$, this last sum converges almost surely to $\sum_{k=1}^{\infty}\left(n_{k}+1\right) \mathbf{1}_{\left\{n_{k} \geq k\right\}} \epsilon_{k}$. Hence $R_{n, j}=O_{P}(1)$. Similarly, $T_{n, j}$ has the same distribution as

$$
\sum_{k=1}^{n} \sum_{t=n-k+1}^{n} \mathrm{e}^{\mathrm{i} t x_{j}} \mathbf{1}_{\left\{n_{k} \geq k\right\}} \epsilon_{k}=\sum_{k=1}^{n} \sum_{u=0}^{k-1} \mathrm{e}^{-\mathrm{i} u x_{j}} \mathbf{1}_{\left\{n_{k} \geq k\right\}} \epsilon_{k} .
$$

If $j / n \rightarrow 0$, this last term converges to $\sum_{k=1}^{\infty} k \mathbf{1}_{\left\{n_{k} \geq k\right\}} \epsilon_{k}$, which is an almost surely finite sum, whence $T_{n, j}=O_{P}(1)$. In conclusion, as long as $j / n \rightarrow 0, W_{n, j}$ is the leading term in the 
decomposition of $D_{n, j}$. Consider now $W_{n, j}$. It can be written as

$$
\begin{aligned}
W_{n, j} & =\sum_{s=1}^{n} \mathrm{e}^{\mathrm{i} s x_{j}} \frac{1-\mathrm{e}^{\mathrm{i}\left(n_{s}+1\right) x_{j}}}{1-\mathrm{e}^{\mathrm{i} x_{j}}} \epsilon_{s}=\sum_{s=1}^{n} \mathrm{e}^{\mathrm{i} s x_{j}} \mathrm{e}^{\mathrm{i} n_{s} x_{j} / 2} \frac{\sin \left(\left(n_{s}+1\right) x_{j} / 2\right)}{\sin \left(x_{j} / 2\right)} \epsilon_{s} \\
& =\sum_{s=1}^{n} \mathrm{e}^{\mathrm{i} s x_{j}} \frac{\sin \left(\left(n_{s}+1\right) x_{j} / 2\right)}{\sin \left(x_{j} / 2\right)} \epsilon_{s}+\sum_{s=1}^{n} \mathrm{e}^{\mathrm{i} s x_{j}}\left(\mathrm{e}^{\mathrm{i} n_{s} x_{j} / 2}-1\right) \frac{\sin \left(\left(n_{s}+1\right) x_{j} / 2\right)}{\sin \left(x_{j} / 2\right)} \epsilon_{s} \\
& =d_{n, j}+\sum_{s=1}^{n} \mathrm{e}^{\mathrm{i} s x_{j}}\left(\frac{\sin \left(\left(n_{s}+1\right) x_{j} / 2\right)}{\sin \left(x_{j} / 2\right)}-n_{s}-1\right) \epsilon_{s} \\
& +\sum_{s=1}^{n} \mathrm{e}^{\mathrm{i} s x_{j}}\left(\mathrm{e}^{\mathrm{i} n_{s} x_{j} / 2}-1\right) \frac{\sin \left(\left(n_{s}+1\right) x_{j} / 2\right)}{\sin \left(x_{j} / 2\right)} \epsilon_{s}=d_{n, j}+r_{n, j} .
\end{aligned}
$$

To deal with the remainder terms, we use the following bounds: there exists a constant $C$ such that for all $u \in \mathbb{R}$ and for all $v \in(0,1)$,

$$
\begin{gathered}
\left|\mathrm{e}^{\mathrm{i} u}-1\right| \leq C(|u| \wedge 1) \\
\left|\frac{\sin (u v)}{\sin (v)}-u\right| \leq C|u|(|u v| \wedge 1)+|u| v^{2} .
\end{gathered}
$$

For $p \in(1, \alpha)$, applying these bounds and the moment bound for independent zero mean random variables with finite $p$-th moment (cf. Petrov (1995), addendum 2.6.20), we have:

$$
\mathbb{E}\left[\left|r_{n, j}\right|^{p}\right] \leq C \sum_{s=1}^{n} \mathbb{E}\left[\left(n_{s}\right)^{p}\left(\left(n_{s} j / n\right) \wedge 1\right)^{p}\right]=C n \mathbb{E}\left[N^{p}((N j / n) \wedge 1)^{p}\right]+C n(j / n)^{2 p} \mathbb{E}\left[N^{p}\right] .
$$

Let us compute $\mathbb{E}\left[N^{p}((N j / n) \wedge 1)^{p}\right]$ for any $p>1$.

$$
\mathbb{E}\left[N^{p}((N j / n) \wedge 1)^{p}\right]=(j / n)^{p} \sum_{k=1}^{n / j} k^{2 p} \mathbb{P}(N=k)+\sum_{k=n / j}^{\infty} k^{p} \mathbb{P}(N=k) \leq C(j / n)^{\alpha-p} L(n) .
$$

Hence, for any $p \in(1, \alpha), \mathbb{E}\left[\left|r_{n, j}\right|\right]=O\left(L(n) n^{1+(1-\alpha) / p} j^{\alpha / p-1}\right)$. If $j \leq n^{\rho}$ for some $\rho \in(0,1-$ $1 / \alpha)$, then $p$ can be chosen close enough to $\alpha$ so that $\lim _{n \rightarrow \infty} h(n) n^{-1 / \alpha} \mathbb{E}\left[\left|r_{n, j}\right|\right]=0$, for any slowly varying function $h$.

Proof of Proposition 4.1 in the case of Taqqu-Levy's process. For clarity, we denote in this proof $x_{n, j}=2 \pi j / n$. By summing over each regime separately, we can express $D_{n, j}$ as

$$
\begin{aligned}
D_{n, j} & =W_{0} \sum_{t=0}^{S_{0}-1} \mathrm{e}^{i t x_{n, j}}+\sum_{k=1}^{M_{n}} W_{k} \sum_{t=S_{k-1}}^{S_{k}-1} \mathrm{e}^{\mathrm{i} t x_{n, j}}+W_{M_{n}+1} \sum_{t=S_{M_{n}}}^{n} \mathrm{e}^{\mathrm{i} t x_{n, j}} \\
& =r_{1, n, j}+w_{M_{n}, n, j}+r_{2, n, j},
\end{aligned}
$$


where we have defined:

$$
\begin{aligned}
r_{1, n, j} & =W_{0} \exp \left\{\mathrm{i}\left(S_{0}-1\right) x_{n, j} / 2\right\} \frac{\sin \left(S_{0} x_{n, j} / 2\right)}{\sin \left(x_{n, j} / 2\right)}, \\
r_{2, n, j} & =W_{M_{n}+1} \exp \left\{\mathrm{i}\left\{S_{M_{n}}+\left(n-S_{M_{n}}\right) / 2\right\} x_{n, j}\right\} \frac{\sin \left(\left\{n-S_{M_{n}}+1\right\} x_{n, j} / 2\right)}{\sin \left(x_{n, j} / 2\right)}, \\
w_{m, n, j} & =\sum_{k=1}^{m} W_{k} \sum_{t=S_{k-1}}^{S_{k}-1} \mathrm{e}^{\mathrm{i} t x_{n, j}}=\sum_{k=1}^{m} \mathrm{e}^{\mathrm{i}\left\{S_{k-1}+\frac{T_{k}-1}{2}\right\} x_{n, j}} \frac{\sin \left(T_{k} x_{n, j} / 2\right)}{\sin \left(x_{n, j} / 2\right)} W_{k} .
\end{aligned}
$$

Obviously, $\left|r_{1, n, j}\right| \leq\left|W_{0}\right| S_{0}$, hence $r_{1, n, j}=O_{P}(1)$, uniformly with respect to $j \leq n / 2$. To deal with $r_{2, n, j}$, note that $n-S_{M_{n}}$ is the forward recurrence time of the stationary renewal process $\left(S_{n}\right)_{n \geq 0}$, hence its marginal distribution is constant and is equal to that of $S_{0}$ (cf. Resnick (1992), Theorem 3.9.1). Thus, for $q<\alpha-1, \mathbb{E}\left[\left|r_{2, n, j}\right|^{q}\right] \leq \mathbb{E}\left[\left|W_{0}\right|^{q}\right] \mathbb{E}\left[S_{0}^{q}\right]<\infty$. $r_{2, n, j}$ is also $O_{P}(1)$, uniformly with respect to $j \leq n / 2$. Applying Lemma A.1, we obtain that $w_{M_{n}, n, j}-w_{[n / \mu], n, j}=$ $o_{P}\left(n^{-1 / \alpha} h(n)\right.$, uniformly with respect to the sequence $j$ and for any slowly varying function $h$. We now prove that $h(n) n^{-1 / \alpha}\left(w_{[n / \mu], n, j}-d_{n, j}\right)=o_{P}(1)$. Define $\tilde{w}_{m, n, j}=\sum_{k=1}^{m} \mathrm{e}^{\mathrm{i}\left(S_{k-1}-1 / 2\right) x_{n, j}}$ $T_{k} W_{k}$. Applying Lemma A.2 with $m=[n / \mu], H(u, v)=\mathrm{e}^{\mathrm{i} u v / 2} \frac{\sin (u v / 2)}{\sin (v / 2)}$ and $\zeta_{n, k}=\mathrm{e}^{\mathrm{i}\left(S_{k-1}-1 / 2\right) x_{n, j}}$, we obtain:

$$
w_{[n / \mu], n, j}-\tilde{w}_{[n / \mu], n, j}=o_{P}\left(n^{1 / \alpha} h(n)\right) .
$$

Define $\hat{w}_{m, n, j}=\sum_{k=1}^{m} \mathrm{e}^{\mathrm{i}\{(k-1) \mu-1 / 2\} x_{n, j}} T_{k} W_{k}$. Applying Lemma A.3 with $\zeta_{k}=T_{k} W_{k}, K(u)=$ $\mathrm{e}^{\mathrm{i} u}$ yields

$$
\tilde{w}_{[n / \mu], n, j}-\hat{w}_{[n / \mu], n, j}=o_{P}\left(n^{1 / \alpha} h(n)\right) .
$$

Finally, we bound $\hat{w}_{[n / \mu], n, j}-d_{n, j}$.

$$
\begin{aligned}
\hat{w}_{[n / \mu], n, j}-d_{n, j} & =\sum_{k=1}^{[n / \mu]}\left(\mathrm{e}^{\mathrm{i} k \mu x_{n, j}} \mathrm{e}^{-\mathrm{i}(\mu+1 / 2) x_{n, j}}-\mathrm{e}^{\mathrm{i} k x_{[n / \mu], j}}\right) \zeta_{k} \\
& =\sum_{k=1}^{[n / \mu]} \mathrm{e}^{\mathrm{i} k \mu x_{n, j}}\left(\mathrm{e}^{-\mathrm{i}(\mu+1 / 2) x_{n, j}}-1\right) \zeta_{k}+\sum_{k=1}^{[n / \mu]}\left(\mathrm{e}^{\mathrm{i} k \mu x_{n, j}}-\mathrm{e}^{\mathrm{i} k x_{[n / \mu], j}}\right) \zeta_{k} .
\end{aligned}
$$

Since $1 /[n / \mu]-1 /(n / \mu)=O\left(n^{-2}\right)$ and $j \leq n^{\rho}$ with $\rho<1-1 / \alpha$, we obtain:

$$
\mathbb{E}\left[\left|\hat{w}_{[n / \mu], n, j}-d_{n, j}\right|\right] \leq C j / n=o\left(n^{1 / \alpha} h(n)\right),
$$

for any slowly varying function $h$.

Proof of Theorem 4.2 in the case of Parke's process. As seen in the proof of Proposition 4.1, the main term in the decomposition of $D_{n, j}$ is $W_{n, j}$, defined in (B.1). To prove convergence to a complex Gaussian law, we use the Wold device. For $a, b \in \mathbb{R}$, denote

$$
\xi_{n, s}(a, b)=\left\{a \cos \left(\left(s+n_{s} / 2\right) x_{j}\right)+b \sin \left(\left(s+n_{s} / 2\right) x_{j}\right)\right\} \frac{\sin \left(\left(n_{s}+1\right) x_{j} / 2\right) \epsilon_{s}}{\sin \left(x_{j} / 2\right)} .
$$


Then $\sum_{s=1}^{n} \xi_{n, s}(a, b)=a \operatorname{Re}\left(W_{n, j}\right)+b \operatorname{Im}\left(W_{n, j}\right)$. Denote $\sigma_{n}^{2}(a, b)=\sum_{s=1}^{n} \mathbb{E}\left[\xi_{n, s}^{2}(a, b)\right]$. To prove that $\sigma_{n}^{-1}(a, b) \sum_{s=1}^{n} \xi_{n, s}(a, b)$ is asymptotically Gaussian, it suffices to prove that

$$
\sum_{s=1}^{n} \mathbb{E}\left[\left|\xi_{n, s}(a, b)\right|^{q}\right]=o\left(\sigma_{n}^{q}(a, b)\right),
$$

for some $q>2$. We first find an equivalent for $\sigma_{n}^{2}(a, b)$. To simplify the notation, without loss of generality, assume $\sigma_{\epsilon}^{2}=1$. We have

$$
\begin{aligned}
\sin ^{2} & \left(x_{j} / 2\right) \mathbb{E}\left[\xi_{n, s}^{2}\right]=\mathbb{E}\left[\left\{a \cos \left(\left(s+n_{s} / 2\right) x_{j}\right)+b \sin \left(\left(s+n_{s} / 2\right) x_{j}\right)\right\}^{2} \sin ^{2}\left(\left(n_{s}+1\right) x_{j} / 2\right)\right] \\
& =a^{2} \mathbb{E}\left[\cos ^{2}\left(\left(s+n_{s} / 2\right) x_{j}\right) \sin ^{2}\left(\left(n_{s}+1\right) x_{j} / 2\right)\right]+a b \mathbb{E}\left[\sin \left(\left(2 s+n_{s}\right) x_{j}\right) \sin ^{2}\left(\left(n_{s}+1\right) x_{j} / 2\right)\right] \\
& +b^{2} \mathbb{E}\left[\sin ^{2}\left(\left(s+n_{s} / 2\right) x_{j}\right) \sin ^{2}\left(\left(n_{s}+1\right) x_{j} / 2\right)\right] \\
& =\frac{a^{2}+b^{2}}{2} \mathbb{E}\left[\sin ^{2}\left(\left(n_{s}+1\right) x_{j} / 2\right)\right]+\frac{a^{2}-b^{2}}{2} \mathbb{E}\left[\cos \left(\left(2 s+n_{s}\right) x_{j}\right) \sin ^{2}\left(\left(n_{s}+1\right) x_{j} / 2\right)\right] \\
& +a b \mathbb{E}\left[\sin \left(\left(2 s+n_{s}\right) x_{j}\right) \sin ^{2}\left(\left(n_{s}+1\right) x_{j} / 2\right)\right] \\
& =\frac{a^{2}+b^{2}}{2} \mathbb{E}\left[\sin ^{2}\left(\left(n_{s}+1\right) x_{j} / 2\right)\right] \\
& +\left\{\frac{a^{2}-b^{2}}{2} \cos \left(2 s x_{j}\right)+a b \sin \left(2 s x_{j}\right)\right\} \mathbb{E}\left[\cos \left(n_{s} x_{j}\right) \sin ^{2}\left(\left(n_{s}+1\right) x_{j} / 2\right)\right] \\
& -\left\{\frac{a^{2}-b^{2}}{2} \sin \left(2 s x_{j}\right)-a b \cos \left(2 s x_{j}\right)\right\} \mathbb{E}\left[\sin \left(n_{s} x_{j}\right) \sin ^{2}\left(\left(n_{s}+1\right) x_{j} / 2\right)\right] .
\end{aligned}
$$

Applying Lemma A.4, we obtain that

$$
\lim _{n \rightarrow \infty} x_{j}^{-\alpha} L\left(1 / x_{j}\right)^{-1} \mathbb{E}\left[h\left(n_{s} x_{j}\right) \sin ^{2}\left(\left(n_{s}+1\right) x_{j} / 2\right)\right]=\alpha \int_{0}^{\infty} h(t) \sin ^{2}(t / 2) t^{-\alpha-1} d t,
$$

with either $h(t)=\cos (t), h(t)=\sin (t)$ or $h(t) \equiv 1$. Now, since $j \rightarrow \infty$, we have:

$$
\left|\frac{1}{n} \sum_{s=1}^{n} \mathrm{e}^{2 \mathrm{i} s x_{j}}\right| \leq \frac{2}{n\left|\mathrm{e}^{2 \mathrm{i} x_{j}}-1\right|}=O\left(j^{-1}\right)=o(1) .
$$

Thus,

$$
\begin{aligned}
& \lim _{n \rightarrow \infty} n^{-1} x_{j}^{2-\alpha} L\left(1 / x_{j}\right)^{-1} \sum_{s=1}^{n} \mathbb{E}\left[\xi_{n, s}^{2}(a, b)\right] \\
&=2 \alpha\left(a^{2}+b^{2}\right) \int_{0}^{\infty} \sin ^{2}(t / 2) t^{-\alpha-1} d t=\left(a^{2}+b^{2}\right) \int_{0}^{\infty} \sin (t) t^{-\alpha} d t \\
& \quad=\left(a^{2}+b^{2}\right) \Gamma(1-\alpha) \sin (\pi(\alpha-1) / 2)=\left(a^{2}+b^{2}\right) \frac{\Gamma(2 H-1)}{2-2 H} \sin (\pi H) .
\end{aligned}
$$

Hence, applying (2.3), we obtain:

$$
\lim _{n \rightarrow \infty}\left(2 \pi n f\left(x_{j}\right)\right)^{-1} \sum_{s=1}^{n} \mathbb{E}\left[\xi_{n, s}^{2}(a, b)\right]=\frac{a^{2}+b^{2}}{2} .
$$


Hence $\sigma_{n}^{2}(a, b) \sim \operatorname{cnf}\left(x_{j}\right) \rightarrow \infty$. Moreover, for any $q>2$, we have:

$$
\begin{gathered}
\mathbb{E}\left[\left|\xi_{n, s}\right|^{q}\right] \leq C(|a|+|b|)^{q} x_{j}^{-q} \mathbb{E}\left[\left|\sin \left(\left(n_{s}+1\right) x_{j} / 2\right)\right|^{q}\right]=O\left(x_{j}^{\alpha-q} L\left(1 / x_{j}\right)\right), \\
\sigma_{n}^{-q}(a, b) \sum_{s=1}^{n} \mathbb{E}\left[\xi_{n, s}^{q}\right]=O\left(\left(n x_{j}^{\alpha}\right)^{1-q / 2}\right) .
\end{gathered}
$$

Since we have assumed that $j \gg n^{1-1 / \alpha}$ and $q>2$, we obtain that $n x_{j}^{\alpha} \rightarrow \infty$ and $\sum_{s=1}^{n} \mathbb{E}\left[\xi_{n, s}^{q}\right]$ $=o\left(\sigma_{n}^{q}(a, b)\right)$ and $(\underline{\mathrm{B} .4})$ holds.

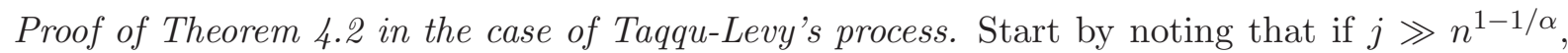
then $\lim _{n \rightarrow \infty}\left(n f\left(x_{n, j}\right)\right)^{-1 / 2} n^{1 / \alpha}=0$. Hence, as in the proof of Proposition 4.1, we obtain that $(2 \pi n)^{-1 / 2} f\left(x_{n, j}\right)^{-1 / 2}\left(D_{n, j}-w_{[n / \mu], n, j}\right)=o_{P}(1)$. We now prove that $w_{[n / \mu], n, j}$ is asymptotically complex Gaussian by the Wold device. Here again, without loss of generality, we assume $\sigma_{W}^{2}=1$. For arbitrary real numbers $a$ and $b$, define $v_{n}^{2}=2 \pi n \sin ^{2}\left(x_{n, j} / 2\right) f\left(x_{n, j}\right)$ and

$$
\begin{aligned}
\eta_{n, k} & =v_{n}^{-1}\left\{a \cos \left(\left(S_{k-1}+\left(T_{k}-1\right) / 2\right) x_{n, j}\right)+b \sin \left(\left(S_{k-1}+\left(T_{k}-1\right) / 2\right) x_{n, j}\right)\right\} \sin \left(T_{k} x_{n, j} / 2\right) W_{k} \\
& \left.\left.=v_{n}^{-1} \cos \left(S_{k-1} x_{n, j}\right)\left\{a \cos \left(\left(T_{k}-1\right) / 2\right) x_{n, j}\right)+b \sin \left(\left(T_{k}-1\right) / 2\right) x_{n, j}\right)\right\} \sin \left(T_{k} x_{n, j} / 2\right) W_{k} \\
& \left.\left.+v_{n}^{-1} \sin \left(S_{k-1} x_{n, j}\right)\left\{-a \sin \left(\left(T_{k}-1\right) / 2\right) x_{n, j}\right)+b \cos \left(\left(T_{k}-1\right) / 2\right) x_{n, j}\right)\right\} \sin \left(T_{k} x_{n, j} / 2\right) W_{k} .
\end{aligned}
$$

Then $(2 \pi n)^{-1 / 2} f^{-1 / 2}\left(x_{n, j}\right)\left\{a \operatorname{Re}\left(w_{[n / \mu], n, j}\right)+b \operatorname{Im}\left(w_{[n / \mu], n, j}\right)\right\}=\sum_{k=1}^{[n / \mu]} \eta_{n, k}$. Denote

$$
\begin{aligned}
B_{1}(u) & =\{a \cos (u / 2)+b \sin (u / 2)\} \sin (u / 2), \\
B_{2}(u) & =\{b \cos (u / 2)-a \sin (u / 2)\} \sin (u / 2), \\
\tilde{\eta}_{n, k} & =v_{n}^{-1}\left\{\cos \left(S_{k-1} x_{n, j}\right) B_{1}\left(T_{k} x_{n, j}\right)+\sin \left(S_{k-1} x_{n, j}\right) B_{2}\left(T_{k} x_{n, j}\right)\right\} W_{k},
\end{aligned}
$$

and $\tilde{w}_{m, n, j}=\sum_{k=1}^{m} \tilde{\eta}_{n, k}$. Then

$$
\sum_{k=1}^{[n / \mu]} \eta_{n, k}-\tilde{\eta}_{n, k}=O_{P}\left(f\left(x_{n, j}\right)^{-1 / 2}\right)=o_{P}(1) .
$$

Define $\mathcal{M}_{j}=\sum_{k=1}^{j} \tilde{\eta}_{n, k}, 1 \leq j \leq[n / \mu]$ and $\mathcal{F}=\left(\mathcal{F}_{k}\right)_{k \geq 1}$ with $\mathcal{F}_{k}=\sigma\left(T_{j}, W_{j}, j \leq k\right)$. Then $\left\{\mathcal{M}_{j}\right\}$ is an $\mathcal{F}$-martingale and $\mathcal{M}_{[n / \mu]}=\tilde{w}_{[n / \mu], n, j}$. Hence, to prove that $\tilde{w}_{[n / \mu], n, j}$ is asymptotically Gaussian, we must prove the conditional Lindeberg conditions:

$$
\begin{aligned}
& \text { there exists } \sigma^{2}>0 \text { such that } \sum_{k=1}^{[n / \mu]} \mathbb{E}\left[\tilde{\eta}_{n, k}^{2} \mid \mathcal{F}_{k-1}\right] \stackrel{P}{\longrightarrow} \sigma^{2}, \\
& \text { and } \forall \epsilon>0, \quad \sum_{k=1}^{[n / \mu]} \mathbb{E}\left[\tilde{\eta}_{n, k}^{2} \mathbf{1}_{\left\{\left|\tilde{\eta}_{n, k}\right| \geq \epsilon\right\}} \mid \mathcal{F}_{k-1}\right] \stackrel{P}{\longrightarrow} 0 .
\end{aligned}
$$


To prove (B.6), note that

$$
\begin{aligned}
\mathbb{E}\left[\tilde{\eta}_{n, k}^{2} \mid \mathcal{F}_{k-1}\right] & =v_{n}^{-2}\left\{\cos ^{2}\left(S_{k-1} x_{n, j}\right) \mathbb{E}\left[B_{1}^{2}\left(T_{1} x_{n, j}\right)\right]\right. \\
& \left.+\sin \left(2 S_{k-1} x_{n, j}\right) \mathbb{E}\left[B_{1}\left(T_{1} x_{n, j}\right) B_{2}\left(T_{1} x_{n, j}\right)\right]+\sin ^{2}\left(S_{k-1} x_{n, j}\right) \mathbb{E}\left[B_{2}^{2}\left(T_{1} x_{n, j}\right)\right]\right\} \sigma_{W}^{2} .
\end{aligned}
$$

Applying Lemmas A.4 and A.5 and using similar computations as in the proof of the previous case, we obtain:

$$
\sum_{k=1}^{[n / \mu]} \mathbb{E}\left[\tilde{\eta}_{n, k}^{2} \mid \mathcal{F}_{k-1}\right] \stackrel{P}{\longrightarrow} \frac{a^{2}+b^{2}}{2} .
$$

To prove (B.7), since $\mathbb{E}\left[\left|W^{q}\right|\right]<\infty$ for some $q>2$, it is sufficient to prove that:

$$
\sum_{k=1}^{[n / \mu]} \mathbb{E}\left[\left|\tilde{\eta}_{n, k}\right|^{q}\right]=o\left(v_{n}^{q}\right)
$$

Since $\mathbb{E}\left[\left|\tilde{\eta}_{n, k}\right|^{q}\right] \leq 2^{q-1} v_{n}^{-q / 2}\left\{\left|B_{1}\left(T_{k} x_{n, j}\right)\right|^{q}+\left|B_{2}\left(T_{k} x_{n, j}\right)\right|^{q}\right\}$ and $\mathbb{E}\left[\left|B_{i}\left(T_{k} x_{n, j}\right)\right|^{q}\right]=O\left(x_{n, j}^{2} f\left(x_{n, j}\right)\right)$, $i=1,2$, we obtain:

$$
\sum_{k=1}^{[n / \mu]} \mathbb{E}\left[\left|\tilde{\eta}_{n, k}\right|^{q}\right]=O\left(n v_{n}^{-q} x_{n, j}^{2} f\left(x_{n, j}\right)\right)=O\left(v_{n}^{1-q / 2}\right)=o(1) .
$$

Hence (B.9) holds. Thus we have shown that $\left\{2 \pi n f\left(x_{n, j}\right\}^{-1 / 2} D_{n, j}\right.$ is asymptotically equivalent to $\left\{2 \pi n f\left(x_{n, j}\right\}^{-1 / 2} w_{[n / \mu], n, j}\right.$ which converges weakly to a standard complex normal law.

Proof of Theorem 5.1. Define $\bar{X}_{n, k}=n^{-1} \sum_{j=1}^{n-k} X_{j}$ and $\tilde{X}_{n, k}=n^{-1} \sum_{j=k+1}^{n} X_{j}$. By Proposition 3.3. $\bar{X}_{n}=O_{P}\left(\ell(n) n^{1 / \alpha-1}\right)$, and obviously, it also holds that $\bar{X}_{n, k}=O_{P}\left(\ell(n) n^{1 / \alpha-1}\right)$ and $\tilde{X}_{n, k}=O_{P}\left(\ell(n) n^{1 / \alpha-1}\right)$. Thus,

$$
\begin{aligned}
\hat{\gamma}_{n}(k) & =n^{-1} \sum_{j=1}^{n-k} X_{j} X_{j+k}-\bar{X}_{n} \tilde{X}_{n, k}-\bar{X}_{n} \bar{X}_{n, k}+\left(\bar{X}_{n}\right)^{2} \\
& =n^{-1} \sum_{j=1}^{n-k} X_{j} X_{j+k}+O_{P}\left(\ell^{2}(n) n^{2 / \alpha-2}\right) .
\end{aligned}
$$

Thus it is sufficient to prove (5.2) for the autocovariances without mean correction. From now on, we denote $\hat{\gamma}_{n}(k)=n^{-1} \sum_{j=1}^{n-k} X_{j} X_{j+k}$ and we pursue the proof in each case separately. 
Proof of Theorem 5.1 in the case of Taqqu-Levy's process.

$$
\begin{aligned}
\hat{\gamma}_{n}(k) & =n^{-1} \sum_{t=1}^{n-k} W_{M_{t}} W_{M_{t+k}}=n^{-1} \sum_{j, j^{\prime}=0}^{\infty} W_{j} W_{j^{\prime}} \sum_{t=1}^{n-k} \mathbf{1}_{\left\{M_{t}=j\right\}} \mathbf{1}_{\left\{M_{t+k}=j^{\prime}\right\}} \\
& =n^{-1} \sum_{j=0}^{\infty} W_{j}^{2} \sum_{t=1}^{n-k} \mathbf{1}_{\left\{M_{t}=M_{t+k}=j\right\}}+n^{-1} \sum_{j \neq j^{\prime}=0}^{\infty} W_{j} W_{j^{\prime}} \sum_{t=1}^{n-k} \mathbf{1}_{\left\{M_{t}=j\right\}} \mathbf{1}_{\left\{M_{t+k}=j^{\prime}\right\}} \\
& =\tilde{\gamma}_{n}(k)+r_{n} .
\end{aligned}
$$

Consider first $r_{n}$. Note that the sums in $j$ and $j^{\prime}$ are limited to $n$ since by definition, $M_{t} \leq t$. If $j^{\prime}<j$ or $j^{\prime}>k$, the event $\left\{M_{t}=j ; M_{t+k}=j^{\prime}\right\}$ is empty. Hence:

$$
\begin{aligned}
\mathbb{E}\left[r_{n}^{2}\right] & =\frac{\sigma_{W}^{4}}{n^{2}} \sum_{j=0}^{\infty} \sum_{j^{\prime}=j+1}^{j+k} \sum_{s, t=1}^{n-k} \mathbb{P}\left(M_{s}=M_{t}=j ; M_{s+k}=M_{t+k}=j^{\prime}\right) \\
& =\frac{\sigma_{W}^{4}}{n^{2}} \sum_{j=0}^{\infty} \sum_{j^{\prime}=j+1}^{j+k} \sum_{t=1}^{n-k} \mathbb{P}\left(M_{t}=j ; M_{t+k}=j^{\prime}\right) \\
& +\frac{\sigma_{W}^{4}}{n^{2}} \sum_{j=0}^{\infty} \sum_{j^{\prime}=j+1}^{j+k} \sum_{1 \leq s<t \leq n-k} \mathbb{P}\left(M_{s}=M_{t}=j ; M_{s+k}=M_{t+k}=j^{\prime}\right)
\end{aligned}
$$

For $s<t$ and $j<j^{\prime}$, the set $\left\{M_{s}=M_{t}=j ; M_{s+k}=M_{t+k}=j^{\prime}\right\}$ is empty if $s+k \leq t$. Hence:

$$
\begin{aligned}
\mathbb{E}\left[r_{n}^{2}\right] & =\frac{\sigma_{W}^{4}}{n^{2}} \sum_{t=1}^{n-k} \mathbb{P}\left(M_{t}<M_{t+k}\right) \\
& +\frac{\sigma_{W}^{4}}{n^{2}} \sum_{s=1}^{n-k-1} \sum_{s+1<t<s+k-1} \mathbb{P}\left(M_{s}=M_{t}<M_{s+k}=M_{t+k}\right)=O\left(n^{-1}\right) .
\end{aligned}
$$

Thus $r_{n}(k)=O_{P}\left(n^{-1 / 2}\right)$. Consider now $\tilde{\gamma}_{n}(k)$. By definition of the renewal process, $M_{t}=$ $M_{t+k}=j$ if and only if $S_{j-1} \leq t<S_{j}$ and $T_{j} \geq k$. Thus

$$
\tilde{\gamma}_{n}(k)=\frac{1}{n} \sum_{j=1}^{M_{n-k}} W_{j}^{2} \sum_{t=1}^{n-k} \mathbf{1}_{\left\{M_{t}=M_{t+k}=j\right\}}=\frac{1}{n} \sum_{j=1}^{M_{n-k}} W_{j}^{2}\left(T_{j}-k\right) \mathbf{1}_{\left\{T_{j} \geq k\right\}} .
$$

Define $\check{\gamma}_{n}(k)=\frac{1}{n} \sum_{j=1}^{[(n-k) / \mu]} W_{j}^{2}\left(T_{j}-k\right) \mathbf{1}_{\left\{T_{j} \geq k\right\}}$. By Lemma A.1 for any slowly varying function $h$, we have that $\check{\gamma}_{n}(k)-\check{\gamma}_{n}(k)=o_{P}\left(n^{1-1 / \alpha} h(n)\right)$. Note now that by definition, $\mathbb{E}\left[\left(T_{1}-\right.\right.$ 
$\left.k) \mathbf{1}_{\left\{T_{1} \geq k\right\}}\right]=\mu \mathbb{P}\left(S_{0} \geq k\right)$. Thus:

$$
\begin{aligned}
\check{\gamma}_{n}(k)-\gamma(k) & =\frac{1}{n} \sum_{j=1}^{[(n-k) / \mu]} W_{j}^{2}\left(T_{j}-k\right) \mathbf{1}_{\left\{T_{j} \geq k\right\}} \\
& =\frac{1}{n} \sum_{j=1}^{[(n-k) / \mu]} W_{j}^{2}\left\{\left(T_{j}-k\right) \mathbf{1}_{\left\{T_{j} \geq k\right\}}-\mathbb{E}\left[\left(T_{1}-k\right) \mathbf{1}_{\left\{T_{1} \geq k\right\}}\right]\right\} \\
& +\frac{\mu \mathbb{P}\left(S_{0} \geq k\right)}{n} \sum_{j=1}^{[(n-k) / \mu]}\left\{W_{j}^{2}-\sigma_{W}^{2}\right\}+\gamma(k)\left\{\mu \frac{[(n-k) / \mu]}{n}-1\right\} \\
& =\frac{1}{n} \sum_{j=1}^{[(n-k) / \mu]} W_{j}^{2}\left\{\left(T_{j}-k\right) \mathbf{1}_{\left\{T_{j} \geq k\right\}}-\mathbb{E}\left[\left(T_{1}-k\right) \mathbf{1}_{\left\{T_{1} \geq k\right\}}\right]\right\}+O_{P}\left(n^{-1 / 2}\right) \\
& =\frac{1}{n} \sum_{j=1}^{[(n-k) / \mu]} W_{j}^{2}\left\{T_{j}-\mathbb{E}\left[T_{1}\right]\right\}+O_{P}\left(n^{-1 / 2}\right) .
\end{aligned}
$$

Thus we conclude that for any slowly varying function $h$,

$$
\hat{\gamma}_{n}(k)-\gamma(k)=\frac{1}{n} \sum_{j=1}^{[n / \mu]} W_{j}^{2}\left\{T_{j}-\mathbb{E}\left[T_{1}\right]\right\}+o_{P}\left(n^{1 / \alpha-1} h(n)\right) .
$$

The rest of the proof is straightforward, given the other proofs in this paper, and is omitted to save space.

Proof of Theorem 5.1 in the case of Parke's process.

$$
\begin{aligned}
\hat{\gamma}_{n}(k) & =n^{-1} \sum_{t=1}^{n-k} \sum_{s \leq t} \sum_{s^{\prime} \leq t+k} g_{s, t} g_{s^{\prime}, t+k} \epsilon_{s} \epsilon_{s^{\prime}}=n^{-1} \sum_{s \leq n-k} \sum_{t=1}^{n-k} \mathbf{1}_{\left\{s \vee 1 \leq t \leq\left(s+n_{s}-k\right) \wedge(n-k)\right\}} \epsilon_{s}^{2} \\
& +n^{-1} \sum_{\substack{s \leq n-k ; s^{\prime} \leq n \\
s \neq s^{\prime}}} \sum_{t=1}^{n-k} \mathbf{1}_{\left\{s \vee 1 \leq t \leq\left(s+n_{s}\right) \wedge(n-k)\right\}} \mathbf{1}_{\left\{\left(s^{\prime}-k\right) \vee 1 \leq t \leq\left(s^{\prime}+n_{s^{\prime}}-k\right) \wedge n\right\}} \epsilon_{s} \epsilon_{s^{\prime}} \\
& =\tilde{\gamma}_{n}(k)+r_{n}(k) .
\end{aligned}
$$


We first consider $r_{n}(k)$. It is split into four terms as follows.

$$
\begin{aligned}
r_{n}(k) & =n^{-1} \sum_{\substack{s \leq 0 ; s^{\prime} \leq k \\
s \neq s^{\prime}}}\left\{\left(s+n_{s}\right)_{+} \wedge\left(s^{\prime}+n_{s^{\prime}}-k\right)_{+} \wedge(n-k)\right\} \epsilon_{s} \epsilon_{s^{\prime}} \\
& +n^{-1} \sum_{s \leq 0} \sum_{s^{\prime}=k+1}^{n}\left[\left\{\left(s+n_{s}\right)_{+} \wedge\left(s^{\prime}+n_{s^{\prime}}-k\right) \wedge(n-k)\right\}-s^{\prime}+k+1\right] \epsilon_{s} \epsilon_{s^{\prime}} \\
& +n^{-1} \sum_{s=1}^{n-k} \sum_{\substack{s^{\prime} \leq k\\
}}\left[\left\{\left(s+n_{s}\right) \wedge\left(s^{\prime}+n_{s^{\prime}}-k\right)_{+} \wedge(n-k)\right\}-s+1\right] \epsilon_{s} \epsilon_{s^{\prime}} \\
& \sum_{\substack{1 \leq s \leq n-k ; k+1 \leq s^{\prime} \leq n \\
s \neq s^{\prime}}}\left[\left(s+n_{s}\right) \wedge\left(s^{\prime}+n_{s^{\prime}}-k\right) \wedge(n-k)-s \vee\left(s^{\prime}-k\right)\right] \epsilon_{s} \epsilon_{s^{\prime}} \\
& =r_{1, n}+r_{2, n}+r_{3, n}+r_{4, n} .
\end{aligned}
$$

By the usual Borel Cantelli argument, $n r_{1, n}$ converges to the almost surely finite sum $\sum_{\substack{s \leq 0 ; t \leq 0 \\ s \neq t+k}}$ $\left\{\left(s+n_{s}\right)_{+} \wedge\left(t+n_{t+k}\right)_{+}\right\} \epsilon_{s} \epsilon_{t+k}$. Hence $r_{1, n}=O_{P}\left(n^{-1}\right)$. By independence of the i.i.d. sequences $\left(\epsilon_{s}\right)$ and $\left(n_{s}\right)$, the terms $r_{2, n}$ and $r_{3, n}$ have the same distribution. We consider for instance the former. Let $S$ be the set of nonpositive integers $s$ such that $s+n_{s} \geq 0$. Then $S$ is almost surely finite. Write $r_{2, n}=n^{-1} \sum_{s \in S} \xi_{n, s} \epsilon_{s}$, with

$$
\xi_{n, s}=\sum_{t=1}^{n-k}\left[\left\{\left(s+n_{s}\right)_{+} \wedge\left(t+n_{t+k}\right) \wedge(n-k)\right\}-t+1\right] \epsilon_{t+k}
$$

For each $s \in S$, we have:

$$
\lim _{n \rightarrow \infty} \xi_{n, s}=\sum_{t=1}^{s+n_{s}}\left[\left\{\left(s+n_{s}\right) \wedge\left(t+n_{t+k}\right)\right\}-t+1\right] \epsilon_{t+k}
$$

Since $S$ is almost surely finite, we thus obtain that

$$
\lim _{n \rightarrow \infty} n r_{2, n}=\sum_{s \in S} \sum_{t=1}^{s+n_{s}}\left[\left\{\left(s+n_{s}\right) \wedge\left(t+n_{t+k}\right)\right\}-t+1\right] \epsilon_{t+k}, \text { almost surely. }
$$

Hence $r_{2, n}=O_{P}\left(n^{-1}\right)$ and similarly $r_{3, n}=O_{P}\left(n^{-1}\right)$. Consider now the last term $r_{4, n}$.

$$
\mathbb{E}\left[r_{4, n}^{2}\right]=\sigma_{\epsilon}^{4} n^{-2} \sum_{\substack{1 \leq s \leq n-k ; 1 \leq t \leq n-k \\ s \neq t+k}} \mathbb{E}\left[\left\{\left(s+n_{s}\right) \wedge\left(t+n_{t+k}\right) \wedge(n-k)-s \vee t\right\}^{2}\right] .
$$

This last expectation is finite, since the term inside is at most $n_{s} \wedge n_{t+k}$, and if $N^{\prime}$ is an independent copy of $N$, then $N \wedge N^{\prime}$ is square integrable. Indeed, we have

$$
\mathbb{P}\left(N \wedge N^{\prime} \geq k\right)=\mathbb{P}(N \geq k)^{2}=L^{2}(k) k^{-2 \alpha} .
$$


Since $L$ is slowly varying, then so is $L^{2}$, and since $\alpha \in(1,2)$, then (B.11) implies that $N \wedge N^{\prime}$ is square integrable. Let us now compute the expectation in the rhs of (B.10). Assume $s<t \leq$ $n-k$.

$$
\begin{aligned}
\mathbb{E}[\{(s+N) \wedge & \left.\left.\left(t+N^{\prime}\right) \wedge(n-k)-s \vee t\right\}^{2}\right] \\
& =\sum_{j=t-s}^{n-k-s} \sum_{j^{\prime}=0}^{n-k-t}\left\{(s+j) \wedge\left(t+j^{\prime}\right)-t\right\}^{2} \mathbb{P}(N=j) \mathbb{P}\left(N^{\prime}=j^{\prime}\right) \\
& =\sum_{j=t-s}^{n-k-s} \sum_{j^{\prime}=0}^{j-t+s} j^{\prime 2} \mathbb{P}(N=j) \mathbb{P}\left(N^{\prime}=j^{\prime}\right) \\
& +\sum_{j=t-s}^{n-k-s} \sum_{j^{\prime}=j-t+s+1}^{n-k-t}(j-t+s)^{2} \mathbb{P}(N=j) \mathbb{P}\left(N^{\prime}=j^{\prime}\right) \leq C L^{2}(t-s)(t-s)^{2-2 \alpha} .
\end{aligned}
$$

Plugging this bound into (B.10), we obtain:

$$
\mathbb{E}\left[r_{4, n}(k)^{2}\right]= \begin{cases}O\left(\tilde{L}(n) n^{2-2 \alpha}\right) & \text { if } \alpha \in(1,3 / 2], \text { with } \tilde{L} \text { slowly varying; } \\ O\left(n^{-1}\right) & \text { if } \alpha \in(3 / 2,2)\end{cases}
$$

In conclusion, we have shown that $r_{n}(k)=O_{P}\left(n^{1-\alpha}\right)$. Consider now $\tilde{\gamma}_{n}(k)$. Still by Borel Cantelli arguments, we have

$$
\begin{aligned}
\tilde{\gamma}_{n}(k) & =n^{-1} \sum_{s \leq 0}\left\{\left(s+n_{s}-k\right)_{+} \wedge(n-k)\right\} \epsilon_{s}^{2} \\
& +n^{-1} \sum_{s=1}^{n-k}\left\{\left(s+n_{s}-k\right) \wedge(n-k)-s+1\right\} \mathbf{1}_{\left\{n_{s} \geq k\right\}} \epsilon_{s}^{2} \\
& =n^{-1} \sum_{s=1}^{n-k}\left(n_{s}-k+1\right) \mathbf{1}_{\left\{n_{s} \geq k\right\}} \epsilon_{s}^{2}+O_{P}\left(n^{-1}\right) .
\end{aligned}
$$

Altogether, we have

$$
\begin{aligned}
& \hat{\gamma}_{n}(k)-\gamma(k)=n^{-1} \sum_{s=1}^{n-k}\left(n_{s}-k+1\right) \mathbf{1}_{\left\{n_{s} \geq k\right\}} \epsilon_{s}^{2}-\gamma(k)+O_{P}\left(n^{1-\alpha}\right) \\
&=n^{-1} \sum_{s=1}^{n-k}\left\{\left(n_{s}-k+1\right) \mathbf{1}_{\left\{n_{s} \geq k\right\}}-\mathbb{E}\left[\left(n_{s}-k+1\right) \mathbf{1}_{\left\{n_{s} \geq k\right\}}\right]\right\} \epsilon_{s}^{2} \\
&+\frac{\mathbb{E}\left[(N-1+k) \mathbf{1}_{\{N \geq k\}}\right]}{n} \sum_{s=1}^{n-k}\left\{\epsilon_{s}^{2}-\sigma_{\epsilon}^{2}\right\}+O_{P}\left(n^{1-\alpha}\right) \\
&=n^{-1} \sum_{s=1}^{n-k}\left\{\left(n_{s}-k+1\right) \mathbf{1}_{\left\{n_{s} \geq k\right\}}-\mathbb{E}\left[\left(n_{s}-k+1\right) \mathbf{1}_{\left\{n_{s} \geq k\right\}}\right]\right\} \epsilon_{s}^{2}+O_{P}\left(n^{-1 / 2}\right)+O_{P}\left(n^{1-\alpha}\right) \\
&=n^{-1} \sum_{s=1}^{n}\left\{n_{s}-\mathbb{E}[N]\right\} \epsilon_{s}^{2}+O_{P}\left(n^{-1 / 2}\right)+O_{P}\left(n^{1-\alpha}\right) .
\end{aligned}
$$


Thus, if $\mathbb{E}\left[\left|\epsilon_{0}\right|^{q}\right]<\infty$ for some $q>2 \alpha$, then $\ell(n)^{-1} n^{1-1 / \alpha}\left(\hat{\gamma}_{n}(k)-\gamma(k)\right)$ converges weakly to an $\alpha$-stable distribution.

\section{References}

[1] Bingham, N.H., Goldie, C.M., and Teugels, J.L. Regular Variation. Encyclopedia of Mathematics and its Applications, 27. Cambridge University Press, Cambridge, 1989.

[2] Bollerslev, T. and Jubinski, D. Equity trading volume and volatility: latent information arrivals and common long-run dependencies. Journal of Business and Economic Statistics 17 (1999), 9-21.

[3] Breiman, L. On some limit theorems similar to the arc-sine law. Theory of Probability and Applications, 10:323-331, 1965.

[4] Chen, W. W. and Hurvich, C.M. Estimating fractional cointegration in the presence of polynomial trends. Journal of Econometrics 117 (2003a), 95-121.

[5] Chen, W. W. and Hurvich, C.M. Semiparametric Estimation of Multivariate Fractional Cointegration. Journal of the American Statistical Association 98 (2003b), 629-642.

[6] Chung, C.-F. Sample Means, Sample Autocovariances, and Linear Regressions of Stationary Multivariate Long Memory Processes. Econometric Theory 18 (2002), 51-78.

[7] Davis, R.A., and Mikosch, T. The sample autocorrelations of heavy-tailed processes with applications to ARCH. Annals of Statistics 26 (1998), 2049-2080.

[8] Deo, R.S. and Hurvich, C.M. On the log periodogram regression estimator of the memory parameter in long memory stochastic volatility models. Econometric Theory, 17, (2001), 686-710.

[9] Deo, R. Hsieh, M. Hurvich, C.M. and Soulier, Ph. Long memory in nonlinear processes. Patrice Bertail, Paul Doukhan and Philippe Soulier. Dependence in Probability and Statistics New York, Springer, (2006).

[10] Embrechts, P., Klüppelberg, C. and Mikosch, T. Modelling Extremal Events for Insurance and Finance. Number 33 in Stochastic modelling and applied probability. Berlin, Springer, 1997.

[11] Geweke, J. and Porter-Hudak, S. The estimation and application of long memory time series models. Journal of Time Series Analysis, 4, (1983), 221-238.

[12] Gorodeckii, V.V. On convergence to semi-stable Gaussian processes. Theory Prob. Appl., 22(3):498-508, 1977. 
[13] Granger C.W.J. and Joyeux R. (1980). An introduction to long memory time series and fractional differencing. J. of Time Series Analysis, 1, 15-30.

[14] Hall, P. and Heyde, C.C. Martingale limit theory and its application. Probability and Mathematical Statistics. New York: Academic Press, 1980.

[15] Harvey, A. C. Long memory in stochastic volatility. In: Knight, J., Satchell, S. (Eds.), Forecasting volatility in financial markets. Butterworth-Heinemann, London, 1998.

[16] Hosking, J.R.M. Fractional differencing. Biometrika 68, (1981), 165-176.

[17] Hurvich, C.M. and Beltrao, K. Asymptotics of the low-frequency ordinates of the periodogram of a long-memory time series, J. of Time Series Analysis 14 (1993), 455-472.

[18] Hurvich, C.M., Deo, R.S., and Brodsky, J. The mean squared error of Geweke and PorterHudak's estimator of the memory parameter of a long-memory time series, J. of Time Series Analysis 19 (1998), 19-46.

[19] Hurvich, C.M., Moulines, E. and Soulier, Ph. The FEXP Estimator for Potentially NonStationary Linear Time Series. Stochastic Processes and their Applications 97 (2002), 307340 .

[20] Hurvich, C.M. and Soulier, Ph. Testing for long memory in volatility. Econometric Theory 18 (2002), 1291-1308.

[21] Ibragimov; I.A. et Linnik, Yu.V. Independent and stationary sequences of random variables Wolters, Groningen, 1971

[22] Johnson, L.W. and Riess, R.D. Numerical Analysis Addison Wesley, Reading, Massachusetts, 1977.

[23] Künsch, H.R. Discrimination between monotonic trends and long-range dependence, J. Applied Probability 23 (1986), 1025-1030.

[24] Künsch, H.R. Statistical aspects of self-similar processes. Proceedings of the World Congress of the Bernoulli Society, Tashkent, Vol. 1, (1987), 67-74.

[25] Lahiri, S.N. A Necessary and Sufficient Condition for Asymptotic Independence of Discrete Fourier Transforms Under Short- and Long-Range Dependence. Annals of Statistics, 31(2), (2003).

[26] Lang, G. and Soulier, Ph. Convergence de mesures spectrales aléatoires et applications à des principes d'invariance. Statistical Inference for Stochastic Processes, 3(1-2), p. 41-51, (2000).

[27] Leipus, R. and Surgailis, D. Random coefficient autoregression, regime switching and long memory. Advances in Applied Probability, 35 (2003), 737-754. 
[28] Liu, M. Modeling long memory in stock market volatility. Journal of Econometrics 99 (2000), 139-171.

[29] Mikosch, T., Resnick, S.I., Rootzen, H., and Stegeman, A. Is network traffic approximated by stable Levy motion or fractional Brownian motion? Annals of Applied Probability, 12:2368, 2002.

[30] Parke, W.R. What is fractional integration? Review of Economics and Statistics 81 (1999), 632-638.

[31] Petrov, V. Limit theorems of probability theory. Oxford University Press, 1995.

[32] Resnick, S.I. Extreme values, regular variation and point processes. New York, Springer, 1987.

[33] Resnick, S.I. Adventures in stochastic processes. Boston, Birkhäuser, 1992.

[34] Robinson, P.M. Log-periodogram regression of time series with long range dependence. Annals of Statistics 23 (1995a), 1043-1072.

[35] Robinson, P.M. Gaussian semiparametric estimation of long range dependence. Annals of Statistics 24 (1995b), 1630-1661.

[36] Taqqu, M.S. Fractional Brownian motion and long-range dependence. Paul Doukhan, Georges Oppenheim and Murad S. Taqqu, editors. Theory and Applications of Long-Range Dependence Boston, Birkhäuser, (2003).

[37] Taqqu, M.S. and Levy, J.B. Using Renewal Processes to Generate Long-Range Dependence and High Variability. Dependence in Probability and Statistics, 73-89. Boston, Birkhauser, 1986.

[38] Terrin, N. and Hurvich, C.M. An Asymptotic Wiener-Ito Representation for the Low Frequency Ordinates of the Periodogram of a Long Memory Time Series. Stochastic Processes and their Applications 54, (1994), 297-307.

[39] Tieslau, M.A., Schmidt, P. and R.T. Baillie. A minimum distance estimator for longmemory processes. Journal of Econometrics 71 (1996), 249-264.

[40] Velasco, C. Non-Gaussian log-periodogram regression Econometric Theory 16 (2000), 4479.

[41] Walker, A.M. Some results concerning the asymptotic Distribution of Sample Fourier Transforms and Periodograms for a Discrete-Time Stationary Process with a Continuous Spectrum. Journal of Time Series Analysis 21, (2000), 95-109.

[42] Yong, C.H. On the asymptotic behavior of trigonometric series. I. Journal of Mathematical Analysis and Applications 33, (1971), 23-34. 